\title{
A Comparative Study of Four Impedance Eduction Methodologies Using Several Test Liners
}

\author{
W. R. Watson* and M. G. Jones ${ }^{\dagger}$ \\ NASA Langley Research Center, Hampton, Virginia 23681-2199
}

\begin{abstract}
A comparative study of four commonly used impedance eduction methods is presented for a range of liner structures and test conditions. Two of the methods are restricted to uniform flow while the other two accommodate both uniform and boundary layer flows. Measurements on five liner structures (a rigid-wall insert, a ceramic tubular liner, a wire mesh liner, a low porosity conventional liner, and a high porosity conventional liner) are obtained using the NASA Langley Grazing Flow Impedance Tube. The educed impedance of each liner is presented for forty-two test conditions (three Mach numbers and fourteen frequencies). In addition, the effects of moving the acoustic source from upstream to downstream and the refractive effects of the mean boundary layer on the wire mesh liner are investigated. The primary conclusions of the study are that 1) more accurate results are obtained for the upstream source, 2) the uniform flow methods produce nearly identical impedance spectra at and below Mach 0.3 but significant scatter in the educed impedance occurs at the higher Mach number, 3) there is better agreement in educed impedance among the methods for the conventional liners than for the rigid-wall insert, ceramic, or wire mesh liner, and 4) the refractive effects of the mean boundary layer on the educed impedance of the wire mesh liner are generally small except at Mach 0.5 .
\end{abstract}

\section{Nomenclature}

$A^{ \pm}, D \quad=$ right and left running mode coefficients, complex polynomial coefficients

$c_{0}, \rho_{0}, M_{0} \quad=$ speed of sound, mean static density, uniform flow Mach number

$d, H, L, W \quad=$ depth of liner cavity, height of duct, length of computational domain, width of duct

$F(\theta, \chi) \quad=$ wall objective function whose global minima occurs at the unknown wall impedance

$f, \omega, k=$ driving frequency, circular harmonic frequency $(2 \pi f)$, freespace wavenumber $\left(\omega / c_{0}\right)$

$G(Z), Z \quad=$ complex polynomial of degree $\mathrm{Q}$, the argument of a complex polynomial

$i,\|\quad\| \quad=$ unit imaginary number $(\sqrt{-1})$, modulus of a complex quantity

$L_{1}, L_{2}, \Delta z \quad=$ axial location of leading edge of liner, axial location of trailing edge of liner, increment spacing

$\kappa, \lambda=$ axial propagation constant in liner, duct eigenvalue in liner

$N, n$ wall, $Q \quad=$ number of modes, number of microphones, number of evenly spaced points in the Prony method

$\{P\},[S] \quad=$ right-hand side vector, coefficient matrix for the Prony method

$\mathcal{P}, \mathcal{U}, \mathcal{V} \quad=$ pressure eigenfunction, axial velocity eigenfunction, transverse velocity eigenfunction

$p, u, v=$ acoustic pressure, axial acoustic particle velocity, and transverse acoustic particle velocity

$x, y, z, t=$ vertical coordinate, horizontal coordinate, axial coordinate, physical time

$\beta, \zeta=$ normalized admittance $(\beta=\sigma+i \gamma)$ of test liner, normalized impedance $(\zeta=\theta+i \chi)$ of test liner

$\theta, \chi, \sigma, \gamma=$ normalized resistance, reactance, conductance, and susceptance of test liner

Subscripts:

$I, n=$ lower wall microphone counter $(I=1,2,3 \ldots$ wwall $)$, duct mode order $(n=0,1,2 \ldots N)$

Num, Meas = a numerically computed quantity, a measured quantity

Superscripts:

,$+-\quad \quad=$ right moving wave, left moving wave

\footnotetext{
* Senior Research Scientist, Research Directorate, Computational AeroSciences Branch, Liner Physics Group, Associate Fellow AIAA

${ }^{\dagger}$ Senior Research Scientist, Research Directorate, Structural Acoustics Branch, Liner Physics Group, Associate Fellow AIAA.
} 


\section{Introduction}

Efficient duct treatments for broadband noise suppression in turbofan engine ducts remain critical to the development of environmentally acceptable commercial aircraft. To this end, an accurate knowledge of duct-treatment impedance in real flows is critical to effective liner design. The acoustic performance of sound absorbing materials in aircraft engines can be greatly affected by the presence of grazing flows and experimental and computational evidence show that significant variation in acoustic performances can occur as the flow Mach number is varied. In the absence of grazing flow, the normal incidence impedance tube is used almost universally for obtaining the acoustic impedance of test liner specimens. However, the geometry of this apparatus (i.e., closed at the sample end) does not lend itself to the grazing flow environment that is typical of liners installed in commercial aircraft engines.

The waveguide methods (the subject of this paper) are operationally convenient for educing liner impedance in a grazing flow environment. Over the last three decades, the NASA Langley Research Center (LaRC) has investigated a number of methods for determining the acoustic impedance of duct liners, ${ }^{1-4}$ with the one based on the convected Helmholtz equation $(\mathrm{CHE})^{3}$ being more widely used. These waveguide methods combine experimental measurements and computation to infer the impedance of the liner and are referred to as impedance eduction techniques. In the first decade of the 21 st century a joint study of available impedance eduction techniques was conducted between NASA and three U.S. companies; General Electric Aircraft Engines, B. F. Goodrich, and Boeing. ${ }^{5-7}$ In this joint study similar liners were tested at NASA and in each company's research facility. The impedance was then educed using each company's impedance eduction methodology, and a comparative study was performed. This comparative study demonstrated the importance of using impedance eduction methods in a complementary manner to improve confidence in educed impedance. It also provided insights into additional advances that were needed to improve the available impedance eduction technology.

Since the time of the joint study described above, three additional impedance eduction techniques have become available in the open literature. These include a method based upon the solution to the linearized Euler equations (LEE), ${ }^{8}$ a method based upon a set of mode-matching equations (MME) ${ }^{9}$ and a straight forward method (SFM). ${ }^{10}$ Although results from each of these methods have been presented for a single liner, a comparative study of these methods has not been performed, nor have they been compared to earlier methods. Further, some of the facilities and eduction methods used during the joint study have been refined and improved. For example, the experimental facilities at LaRC have been redesigned to allow more modularity in test setup and to avoid having modes cut on in more than one direction at the same frequency. The CHE method now uses multiple starting values for the initial impedance (to improve robustness) and the exit impedance boundary condition has been replaced with an exit pressure boundary condition (to improve accuracy). The single mode method (SMM) ${ }^{5}$ used in the joint study was applicable only to unidirectional, single mode sound fields. However, the SMM used in this paper is applicable to more complicated sound fields because the required inputs have been calculated using the Prony method. ${ }^{10}$ Also, at the 18th CEAS/AIAA aeroacoustics conference in Colorado Spings, Colorado, the need for a comparative study of impedance eduction techniques was emphasized.

The purpose of this paper is to perform a comparative study of impedance eduction techniques for a range of liner structures and test conditions. Acoustic pressure measurements on five liner structures (a rigid-wall insert, a ceramic tubular liner, a wire mesh liner, a low porosity conventional liner, and a high porosity conventional liner) are obtained using the NASA Langley Grazing Flow Impedance Tube (GFIT). The impedance of each liner is then educed with identical test data using, 1) the LEE method, 2) the latest version of the CHE method, 3) a non-progressive wave field version of the SMM, and 4) the SFM. A comparative study of the educed impedance is then presented for fortytwo test conditions (three Mach numbers and fourteen frequencies). In addition, the effects of the mean boundary layer and of moving the acoustic source from upstream (the exhaust mode configuration) to downstream (the inlet mode configuration) are investigated. The paper is organized into five sections. Section II introduces the measurement apparatus, the NASA Langley GFIT. Section III introduces the impedance eduction techniques and describes other new features that were specifically implemented in the current study. Section IV presents a description of the test liners as well as the most significant results of the comparative study. The primary conclusions of the paper are presented in section V.

\section{The Measurement Apparatus}

Measured data needed to perform the current study are acquired in the NASA Langley GFIT. A sketch of this stateof-the-art research facility is shown in Fig. 1. The GFIT has a rectangular cross-sectional geometry that is designed so that higher-order modes in the horizontal and vertical dimensions cut on at different frequencies. It allows convenient 


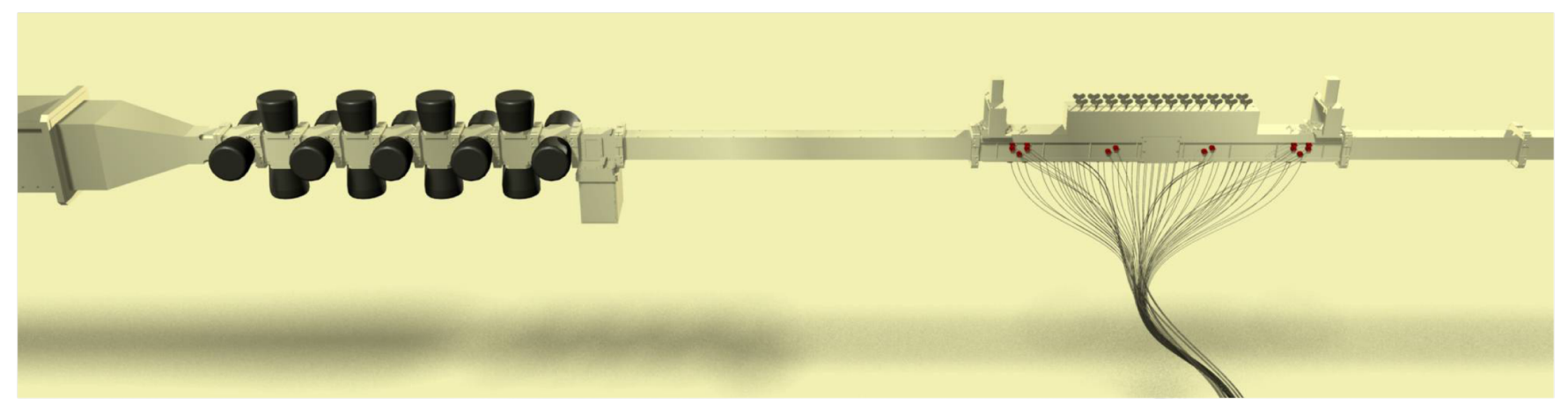

Figure 1. Sketch of the LaRC grazing flow impedance tube (GFIT) and instrumentation.

evaluation of acoustic liners with lengths up to $610 \mathrm{~mm}$. As shown in Fig. 1, flow propagates from left to right through the GFIT. High-pressure air is supplied on the upstream end and a vacuum blower is located on the downstream end, such that the test window containing the acoustic liner is exposed to near-ambient pressure conditions for mean flows up to Mach 0.6. The surface of the test liner forms a portion of the upper wall of the flow duct and as depicted in Fig. 1, the source section consists of up to eighteen acoustic drivers. The acoustic drivers can be mounted upstream (exhaust mode) or downstream (inlet mode) of the test section, and are used to generate tones (one frequency at a time) at up to $150 \mathrm{~dB}$ over a frequency range of 0.4 to $3.0 \mathrm{kHz}$. Figure 2 shows a sketch of the portion of the flow duct that

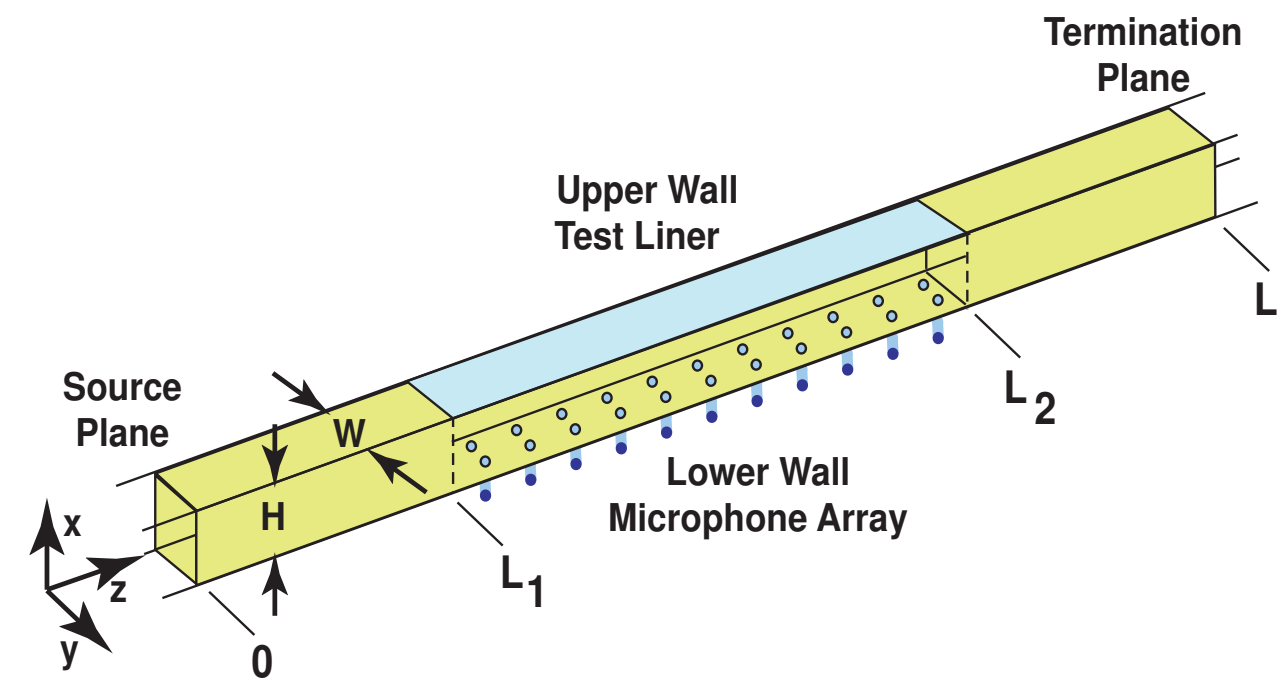

Figure 2. Schematic of the LaRC grazing flow impedance tube (GFIT) flow duct.

is used as the computational domain, and provides the coordinate system for which impedance eduction analyses are performed. The computational domain has a height (i.e., $H$ ) of $63.5 \mathrm{~mm}$, a width (i.e., $W$ ) of $50.8 \mathrm{~mm}$, and a length (i.e., $L$ ) of $1016 \mathrm{~mm}$. As shown in Fig. 2, the surface of the test liner forms a portion of the upper wall for $L_{1} \leq z \leq L_{2}$ and the remainder of the duct consists of rigid walls. It should be noted that the location of the leading (i.e., $z=L_{1}$ ) and trailing (i.e., $z=L_{2}$ ) edges of the liner may vary (e.g., different liner lengths). The test liner is assumed to have an unknown, but uniform, normalized impedance, $\zeta$. Throughout this paper all impedances are normalized with the characteristic impedance, $\rho_{0} c_{0}$, of the air flowing in the duct. Fifty-three microphones flush-mounted in the lower wall (opposite the liner) are used to measure the acoustic pressure field over the axial extent of $0 \leq z \leq L$ (see Fig. 2), so that the impedance eduction methods described in the following section can be used to determine the impedance of the test liner. For each data acquisition, 2000 averages on each microphone channel (blocks of 2048 data points per average) are taken. To reduce the influence of flow noise, a cross-spectrum signal extraction method is used to determine the amplitudes and phases at each of the microphone locations relative to the amplitude and phase at the reference 
microphone location. A dual-axis traverse probe system is used to measure the flow profile at selected axial planes in the GFIT. Two pitot probes are used for this measurement. One is shaped to allow detailed measurements close to the upper wall, and the other is shaped to allow corresponding measurements near the lower wall. The traverse system uses two stepping motors to successively move the pitot probe to preselected measurement locations, such that the full flow profile can be accurately determined. This system is used to acquire flow profiles upstream and downstream of the liner, from which a representative average flow Mach number is computed and then used as the uniform flow Mach number for the impedance eduction methodologies. Additionally, the measured mean flow Mach number profile at the midspan of the GFIT is used to simulate the sheared flow effects. The probes that measure the mean flow profiles are removed for subsequent acoustic measurements.

\section{Impedance Eduction Methodologies}

Each impedance eduction technique discussed in this section is described elsewhere. Therefore, only enough detail is presented to highlight new features that are implemented in the current study and to provide clarity and continuity with the Results section. Some of the methods assume uniform mean flow whereas others may be implemented in a uniform or boundary layer flow. Three other important simplifying assumptions that are used in describing each impedance eduction methodology are: 1) the time dependence is of the form $\left.e^{i \omega t}, 2\right)$ the source frequency is sufficiently low so that only plane waves are present at the source and exit planes of the computational domain, and 3) the test liner is locally reacting (i.e., acoustic waves propagating in and out of the liner surface are normal to the surface) so that the Myers locally reacting wall impedance boundary condition ${ }^{11}$ is applicable. Although, there is no guarantee that the lowest order mode is a plane wave when a shear flow is present, previous tests with microphones located around the periphery of the source and exit plane shows that the plane wave assumption is reasonable over the frequency range of interest.

\section{A. The Linearized Euler Equations (LEE)}

The LEE method of impedance eduction ${ }^{8}$ is based on the solution to the linearized Euler equations and has the advantage that it can accommodate a uniform or boundary layer flow. The three equations representing the linearized conservation equations for mass, axial momentum, and transverse momentum, respectively, in a parallel, shear, mean flow are: ${ }^{8}$

$$
\begin{gathered}
i k p+M_{0} \frac{\partial p}{\partial z}=-\rho_{0} c_{0}\left(\frac{\partial u}{\partial z}+\frac{\partial v}{\partial x}\right) \\
i k u+M_{0} \frac{\partial u}{\partial z}+\frac{d M_{0}}{d x} v=-\frac{1}{\rho_{0} c_{0}} \frac{\partial p}{\partial z} \\
i k v+M_{0} \frac{\partial v}{\partial z}=-\frac{1}{\rho_{0} c_{0}} \frac{\partial p}{\partial x}
\end{gathered}
$$

where homentropic flow is assumed (i.e., $p=c_{0}^{2} \rho$ ) and only plane waves are assumed in the spanwise direction (i.e., the $y$ direction).

The first lower wall microphone (not shown in Fig. 2) is located upstream of the liner at the source plane (i.e, $z=0)$ and is used to measure the source plane pressure $(p(0,0)$ ), whereas the last microphone (microphone \#53) is located at the exit plane $(z=L)$ and is used to measure the exit plane acoustic pressure $(p(L, 0))$. Note that the last microphone is also not shown in Fig. 2. Because the source and exit plane acoustic pressure is planar, it is convenient to use the measured acoustic pressures at the source and exit planes as the boundary conditions

$$
\begin{aligned}
& p(0, x)=p(0,0) \\
& p(L, x)=p(L, 0)
\end{aligned}
$$

In addition, the linearized Euler equations require that one velocity component be specified at the source plane. Because the source plane pressure is assumed to be a plane wave, we specify the transverse component of the acoustic particle velocity vector (i.e., this component of the acoustic velocity is zero for a plane wave)

$$
v(0, x)=0
$$


The lined portion of the upper wall of the GFIT is assumed locally reacting. The proper wall impedance boundary condition in this case has been derived by Myers ${ }^{11}$

$$
v(H, z)=\left(1+\frac{M_{0}}{i k} \frac{\partial}{\partial z}\right)\left[\frac{p(H, z)}{\rho_{0} c_{0} \zeta}\right]
$$

Here, the wall admittance $(1 / \zeta)$ is taken as zero for the rigid wall portion of the upper wall. However, along the test liner portion of the upper wall, the wall impedance is set to the uniform impedance, $\zeta$, of the test liner.

The LEE method of impedance eduction solves (1)-(7) using a cubic finite element method (FEM) with the lower and upper wall boundary conditions imposed in strong form (i.e., by constraining the nodal values of $v$ and $p$ ). The unknown normalized impedance is educed by an iterative process that determines the resistance, $\theta$, and reactance, $\chi$, that reproduces the acoustic wall pressures measured by the lower wall microphones (see Fig. 2). This is achieved by minimizing the objective function

$$
F(\theta, \chi)=\sum_{I=1}^{n w a l l}\left\|\left[\left.p\left(z_{I}, 0\right)\right|_{\text {Num }}-\left.p\left(z_{I}, 0\right)\right|_{\text {Meas }}\right]\right\|
$$

where $n$ wall is the number of microphones (i.e., 53 for this investigation). The minimization is achieved using Stewart's adaptation of the Davidon-Fletcher-Powell (SDFP) optimization algorithm. ${ }^{13}$ SDFP returns the normalized resistance, $\theta$, and normalized reactance, $\chi$, of the test liner.

\section{B. The Convected Helmholtz Equation (CHE)}

The CHE method of impedance eduction ${ }^{3}$ was introduced in 1999. The acoustic field propagating through the flow duct (see Fig. 2), under the assumed condition of uniform mean flow (with plane waves assumed in the spanwise direction) satisfies a convected Helmholtz equation on the acoustic pressure field

$$
\left(1-M_{0}^{2}\right) \frac{\partial^{2} p(z, x)}{\partial z^{2}}+\frac{\partial^{2} p(z, x)}{\partial x^{2}}-2 i k M_{0} \frac{\partial p(z, x)}{\partial z}+k^{2} p(z, x)=0
$$

The boundary condition at the rigid lower wall is that the normal component of the acoustic particle velocity must vanish. When written in terms of the acoustic pressure field this boundary condition is

$$
\frac{\partial p(z, 0)}{\partial x}=0
$$

Just as with the LEE method, it is convenient to use the measured acoustic pressures at the source and exit planes as the boundary conditions

$$
\begin{aligned}
& p(0, x)=p(0,0) \\
& p(L, x)=p(L, 0)
\end{aligned}
$$

The locally-reacting wall impedance boundary condition (when written in terms of the acoustic pressure field) is ${ }^{11}$

$$
-\frac{\partial p(z, H)}{\partial x}=i k\left(\frac{p(z, H)}{\zeta}\right)+2 M_{0} \frac{\partial}{\partial z}\left(\frac{p(z, H)}{\zeta}\right)+\frac{M_{0}^{2}}{i k} \frac{\partial^{2}}{\partial z^{2}}\left(\frac{p(z, H)}{\zeta}\right)
$$

where $1 / \zeta$ is taken as zero along the rigid wall portion of the upper wall. The CHE method of impedance eduction solves the boundary value problem defined in (9)-(13) using a finite element method (FEM). The impedance boundary conditions are incorporated in the FEM analysis in weak form and cubic Hermite polynomials are used as the basis functions. The unknown normalized impedance, $\zeta$, is educed by minimizing the objective function defined in (8).

\section{The Single Mode Method (SMM)}

The single mode method for impedance eduction ${ }^{1}$ was first presented in 1976 for uniform flows and was later extended to non-uniform flows. ${ }^{4}$ However, the original non-uniform flow method ${ }^{4}$ was restricted to sound fields in the liner test section that were free from end reflections or multi-modal acoustic contamination. With the addition of more sophisticated data analysis techniques ${ }^{10}$ the SMM can now be applied to nonprogressive sound fields that are contaminated with multi-modal acoustic effects. It is possible to perform a normal mode analysis (in a uniformly lined section of 
duct) with non-uniform mean flows by numerically integrating a second-order differential equation. This ordinary differential equation was first derived and solved for rigid wall ducts using asymptotic methods ${ }^{14}$ and was later extended to ducts with sound absorbing materials. ${ }^{15}$ To the author's knowledge, the current effort represents the first attempt to use the equation to educe impedance in a sound field containing end reflections and multi-modal acoustic effects. The normal mode analysis assumes that the acoustic field can be expressed in terms of the product of a normal duct mode and a complex exponential

$$
p(z, x)=\mathscr{P}_{n}(x) e^{-i \kappa_{n} z}, \quad u(z, x)=\mathcal{U}_{n}(x) e^{-i \kappa_{n} z}, \quad v(z, x)=\mathcal{V}_{n}(x) e^{-i \kappa_{n} z}
$$

Substituting the above normal mode solution into the linearized Euler equations (Eqs. (1)-(3)) and eliminating the acoustic particle velocities gives a single ordinary differential equation on the acoustic pressure mode

$$
\mathcal{P}_{n}^{\prime \prime}+\left[\frac{2 \kappa_{n}}{c_{0}\left(k-\kappa_{n} M_{0}\right)}\left(c_{0} M_{0}\right)^{\prime}\right] \mathcal{P}_{n}^{\prime}+\left[\left(k-\kappa_{n} M_{0}\right)^{2}-\kappa_{n}^{2}\right] \mathcal{P}_{n}=0
$$

The wall boundary conditions are

$$
\begin{gathered}
\mathcal{P}_{n}^{\prime}(0)=0 \\
\mathcal{P}_{n}^{\prime}(H)=-i k\left(1-\frac{\kappa_{n} M_{0}(H)}{k}\right)^{2} \frac{\mathcal{P}_{n}(H)}{\zeta}
\end{gathered}
$$

Given $\kappa_{n}$ and the mean flow Mach number profile, $M_{0}(x),(15)$-(17) can be solved to obtain the wall impedance, $\zeta$. No exact solution for the impedance satisfying equations (15)-(17) has been found for a general Mach number profile, $M_{0}(x)$. However, the impedance can be determined numerically, provided that critical layers (i.e., locations at which $\left.k-\kappa_{n} M_{0}(x)=0\right)$ do not exist. Because the boundary value problem for the normal modes is homogeneous, one is free to arbitrarily specify the value of the pressure mode at the lower wall to obtain a second condition at the lower wall. Thus, (15)-(17) can be solved numerically as an initial-value problem to obtain the impedance of the upper wall. The procedure is summarized in the following four-step process

1. Extract an axial propagation constant, $\kappa_{n}$ from the measured lower wall acoustic pressure profile

2. Integrate (15) numerically from the lower to the upper wall of the duct starting with the conditions $\mathcal{P}_{n}(0)=1$, and $\mathcal{P}_{n}^{\prime}(0)=0$

3. At the upper wall obtain $\mathcal{P}_{n}(H)$ and $\mathcal{P}_{n}^{\prime}(H)$

4. Apply (17) to obtain the wall impedance

$$
\zeta=-i k\left(1-\frac{\kappa_{n} M_{0}(H)}{k}\right)^{2} \frac{\mathcal{P}_{n}(H)}{P_{n}^{\prime}(H)}
$$

The numerical method implemented to integrate (15) in this work used a sequence of steps in the independent variable, $x$. An adaptive procedure determines the size of each of these steps. In general, if the solution is varying rapidly over a particular portion of the transverse coordinate, the numerical procedure reduces the step size to accurately track the solution. This adaptive procedure is therefore able to obtain an accurate solution when $M_{0}(x), \kappa_{n}$, and $k$ are such that the differential equation is extremely stiff.

\section{The Straight Forward Method (SFM)}

The straight forward method of impedance eduction was developed in a recent paper. ${ }^{10}$ To begin, the closed-form solution for the acoustic pressure field, $p(z, x)$, within the liner test section under the assumed condition of uniform mean flow is

$$
p(z, x)=\sum_{n=0}^{N}\left[A_{n}^{+} e^{-i \kappa_{n}^{+} z} \cos \left(\lambda_{n}^{+} x\right)+A_{n}^{-} e^{-i \kappa_{n}^{-} z} \cos \left(\lambda_{n}^{-} x\right)\right]
$$

Equation (19) is also the closed-form solution in the rigid-wall sections provided that

$$
\begin{gathered}
\lambda_{n}^{ \pm}=\frac{n \pi}{H} \quad(n=0,1,2, \ldots \infty) \\
\frac{\kappa_{n}^{\mp}}{k}=\frac{M_{0} \pm \sqrt{1-\left(1-M_{0}^{2}\right)[(n \pi /(k H))]^{2}}}{-\left(1-M_{0}^{2}\right)}
\end{gathered}
$$


To obtain an exact expression for the test liner impedance, substitute (19) into the Myers wall impedance boundary conditions (13) to obtain

$$
\zeta=-\frac{i k\left(1-M_{0} \kappa_{n}^{ \pm} / k\right)^{2}}{\lambda_{n}^{ \pm} \tan \left(\lambda_{n}^{ \pm} H\right)}
$$

In the straight forward method of impedance eduction the axial propagation constant, $\kappa_{n}^{ \pm}$, is measured using the Prony method ${ }^{10}$ and the dispersive relation

$$
\left(\lambda_{n}^{ \pm}\right)^{2}=k^{2}-2 k M_{0} \kappa_{n}^{ \pm}-\left(1-M_{0}^{2}\right)\left(\kappa_{n}^{ \pm}\right)^{2}
$$

is then used to compute the duct eigenvalue (i.e., $\lambda_{n}^{ \pm}$). Given $\kappa_{n}^{ \pm}$and $\lambda_{n}^{ \pm}$, the unknown liner impedance is then extracted using the closed form expression given in (22).

To obtain a measurement of $\kappa_{n}^{ \pm}$using the Prony method, one first truncates the series in (19) to a total of $Q$ modes and measures the acoustic pressure field at $2 Q$ evenly spaced points, $z_{J}$, along the lower wall. Prony's method is then used to extract the axial wavenumbers from the measured lower wall pressure measurements. The procedure is to first solve the matrix equation

$$
[S]=\left[\begin{array}{ccccc}
P_{0} & P_{1} & P_{2} & \cdots & P_{Q-1} \\
P_{1} & P_{2} & P_{3} & \cdots & P_{Q} \\
P_{2} & P_{3} & P_{4} & \cdots & P_{Q+1} \\
P_{3} & P_{4} & P_{5} & \cdots & P_{Q+2} \\
\vdots & \vdots & \vdots & \vdots & \vdots \\
P_{Q-1} & P_{Q} & P_{Q+1} & \cdots & P_{2 Q-2}
\end{array}\right],\{D\}=\left\{\begin{array}{c}
D_{1} \\
D_{2} \\
D_{3} \\
\vdots \\
D_{Q}
\end{array}\right\},\{P\}=\left\{\begin{array}{c}
P_{Q} \\
P_{Q+1} \\
P_{Q+2} \\
\vdots \\
P_{2 Q-1}
\end{array}\right\}
$$

where, $P_{J}=p\left(z_{J}, 0\right)$. Equation (24) is a linear system of equations containing $Q$ equations and $Q$ unknowns. Equation (24) is solved using a standard linear equation solver. The solution to (24) gives the polynomial coefficients, $D_{1}, D_{2}, D_{3}, \ldots D_{Q}$ and the measured $Q$ axial wavenumbers are determined from the relation

$$
\kappa_{n}=\frac{\log _{e}\left(Z_{n}\right)}{-i \Delta z}(n=1,2, \ldots Q)
$$

Here $Z_{n}$ are the complex roots of the $Q$ th degree polynomial, $G\left(Z_{n}\right)=0$, where

$$
G(z)=D_{1}+D_{2} z+D_{3} z^{2}+D_{4} z^{3}+\ldots+D_{Q} z^{Q-1}+z^{Q}
$$

The straight forward method of impedance eduction is amazingly simple. It is worth noting however, that the straight forward method of impedance eduction is only a simplified version of the SMM. The SFM is obtained from the SMM as follows: 1) use the Prony method to extract the axial propagation constant, $\kappa_{n}^{ \pm}$, and 2) substitute the closed-form solution for the acoustic pressure mode (i.e., $\mathcal{P}_{n}^{ \pm}(x)=\cos \left(\lambda_{n}^{ \pm} x\right)$ ) into (18) to obtain the unknown impedance.

\section{Results and Discussion}

In this section, the impedance eduction methods are tested both with uniform and boundary layer flows. First the methods are tested utilizing data synthesized from the exact mode solution for uniform flow (19). This affords one the opportunity to test the impedance eduction methodologies when they are free from measurement uncertainties. Following the investigation using synthesized data, the methodologies are compared using measured data that not only contain measurement uncertainties but realistic boundary layer effects. The effects of the boundary layer are only investigated for the wire mesh liner because detail mean flow profiles measurements were only available for this liner. Also, in the CHE and LEE impedance eduction methodology, SDFP is initialized using an initial normalized resistance of 0.5 (i.e., $\theta=0.5$ ) and an initial normalized reactance of -0.5 (i.e., $\chi=-0.5$ ). Further, the normalized resistance and reactance in SDFP are constrained so that $0.0 \leq \theta \leq 10.0$ and $-10.0 \leq \chi \leq 10.0$. These limits on $\theta$ and $\chi$ are typical of those used in aircraft for engine noise reduction.

Each impedance eduction technique is first tested on synthesized data obtained from the exact mode solution for right-running and left-running waves in the GFIT. Results are obtained for a rigid wall and a uniformly lined (i.e., liner extending from $z=0$, to $z=L$ ) duct with uniform flow Mach numbers of 0.0, 0.3, and 0.5. Only the lowest order mode 
(i.e., $n=0)$ is allowed to propagate down the duct. The lower wall acoustic pressure profile, $\left.p\left(z_{I}, 0\right)\right|_{\text {Meas }}$, needed for the impedance eduction (see Eq. (8) ) is obtained using the exact mode solution given in (19) for the soft wall duct, and the solution in (20) and (21) for the rigid wall duct. For both the exhaust and inlet mode simulations, these exact solutions are used to obtain the synthesized data. In the exhaust mode simulation, to obtain the source plane boundary condition, the lower wall acoustic pressure profile, and the exit plane boundary condition, the complex wave coefficient of the left running wave is set to zero $\left(A_{n}^{-}=0\right)$ and the mode coefficient of the right running wave is set to a $130 \mathrm{~dB}$ sound pressure level (SPL). For the inlet mode simulation, the complex wave coefficient of the right-running wave is set to zero $\left(A_{n}^{+}=0\right)$ and the mode coefficient of the left-running wave is set to an SPL of $130 \mathrm{~dB}$. These two example problems, therefore, test the ability of each eduction procedure to converge to the correct admittance of a rigid wall duct $(\beta=0.0+0.0 i)$ and a soft wall $(\zeta=\theta+i \chi)$ when there are no measurement uncertainties in the data and all assumptions of the eduction model are satisfied. The FEM codes (LEE and CHE) were run with 13 evenly spaced cubic elements in the $x$ direction and 81 evenly spaced elements in the axial direction of the duct. Doubling of the spatial grid (i.e., 26 evenly spaced elements in the $x$ direction and 162 evenly spaced elements in the axial direction) produced changes in the acoustic impedance only in the third decimal digit of precision.

\section{A. Synthesized Data}

Table 1. Normalized wall impedance, $\zeta$, and axial propagation constant, $\kappa_{0}^{+}$, of right-running wave in liner test section.

\begin{tabular}{|c|c|l|l|l|c|c|}
\hline & \multicolumn{2}{|c|}{$M_{0}=0.0$} & \multicolumn{2}{c|}{$M_{0}=0.3$} & \multicolumn{2}{c|}{$M_{0}=0.5$} \\
\hline$f$ & $\zeta$ & $\kappa_{0}^{+}$ & $\zeta$ & $\kappa_{0}^{+}$ & $\zeta$ & $\kappa_{0}^{+}$ \\
\hline 0.4 & $0.166-3.713 \mathrm{i}$ & $10.267-1.300 \mathrm{i}$ & $0.949-1.938 \mathrm{i}$ & $7.213-0.606 \mathrm{i}$ & $1.252-0.963 \mathrm{i}$ & $6.022-0.416 \mathrm{i}$ \\
\hline 0.6 & $0.082-2.298 \mathrm{i}$ & $14.197-1.173 \mathrm{i}$ & $0.768-2.067 \mathrm{i}$ & $10.175-0.557 \mathrm{i}$ & $0.996-2.092 \mathrm{i}$ & $8.571-0.386 \mathrm{i}$ \\
\hline 0.8 & $0.119-1.487 \mathrm{i}$ & $19.622-2.403 \mathrm{i}$ & $0.605-1.346 \mathrm{i}$ & $13.854-1.026 \mathrm{i}$ & $0.883-1.047 \mathrm{i}$ & $11.615-0.691 \mathrm{i}$ \\
\hline 1.0 & $0.130-0.968 \mathrm{i}$ & $23.648-5.189 \mathrm{i}$ & $0.731-0.939 \mathrm{i}$ & $17.076-2.126 \mathrm{i}$ & $1.007-0.747 \mathrm{i}$ & $14.369-1.408 \mathrm{i}$ \\
\hline 1.2 & $0.123-0.588 \mathrm{i}$ & $26.018-7.845 \mathrm{i}$ & $0.808-0.693 \mathrm{i}$ & $19.799-3.309 \mathrm{i}$ & $1.146-0.897 \mathrm{i}$ & $16.821-2.181 \mathrm{i}$ \\
\hline 1.4 & $0.273-0.176 \mathrm{i}$ & $27.830-10.384 \mathrm{i}$ & $0.776-0.537 \mathrm{i}$ & $22.534-4.495 \mathrm{i}$ & $1.037-0.662 \mathrm{i}$ & $19.315-2.929 \mathrm{i}$ \\
\hline 1.6 & $0.146+0.019 \mathrm{i}$ & $26.638-10.049 \mathrm{i}$ & $0.813-0.314 \mathrm{i}$ & $23.815-5.940 \mathrm{i}$ & $1.101-0.471 \mathrm{i}$ & $21.002-4.007 \mathrm{i}$ \\
\hline 1.8 & $0.122+0.271 \mathrm{i}$ & $28.244-7.054 \mathrm{i}$ & $0.859-0.111 \mathrm{i}$ & $24.315-5.850 \mathrm{i}$ & $1.281-0.369 \mathrm{i}$ & $21.986-4.422 \mathrm{i}$ \\
\hline 2.0 & $0.124+0.527 \mathrm{i}$ & $31.995-4.492 \mathrm{i}$ & $0.935+0.148 \mathrm{i}$ & $25.809-4.123 \mathrm{i}$ & $1.394-0.326 \mathrm{i}$ & $23.027-3.521 \mathrm{i}$ \\
\hline 2.2 & $0.163+0.801 \mathrm{i}$ & $36.189-3.573 \mathrm{i}$ & $1.046+0.272 \mathrm{i}$ & $28.705-3.239 \mathrm{i}$ & $1.407-0.343 \mathrm{i}$ & $25.351-2.795 \mathrm{i}$ \\
\hline 2.4 & $0.218+0.994 \mathrm{i}$ & $40.648-2.826 \mathrm{i}$ & $1.286+0.476 \mathrm{i}$ & $31.913-2.409 \mathrm{i}$ & $1.407-0.343 \mathrm{i}$ & $27.974-2.047 \mathrm{i}$ \\
\hline
\end{tabular}

For the rigid-wall duct in the exhaust mode configuration, we use the exact solution for the lowest order rightrunning axial propagation constant, $\kappa_{0}^{+}=k /\left(1+M_{0}\right)$. However, in the inlet mode configuration we use the exact solution for the lowest order left-running axial propagation constant, $\kappa_{0}^{-}=-k /\left(1-M_{0}\right)$. The normalized resistance and reactance spectrum used to calculate the axial propagation constant, $\kappa_{0}^{+}$, for each Mach number in the soft wall duct are tabulated to three decimal digits of precision in Table 1 . Values of $\kappa_{0}^{+}$corresponding to this impedance spectrum are also computed in Table 1. Note that at the highest Mach number (Mach 0.5), plane wave propagation is not supported at frequencies above $2.4 \mathrm{kHz}$. Therefore the results in the table have been terminated at $2.4 \mathrm{kHz}$. Also Table 1 is only for the exhaust configuration, similar data were computed for the inlet configuration in the soft wall duct. The method used to calculate the axial propagation constants in the soft wall duct was identical to that used in a previous paper. ${ }^{16}$

Given the axial propagation constants for the rigid and soft wall portions of duct, each impedance eduction method was run and the impedance that produced the input data was educed to determine if it matched a zero admittance (for the rigid wall) and the impedance spectrum tabulated in Table 1 (for the soft wall duct). Each of the four impedance eduction methodologies (LEE, CHE, SMM, and SFM) educed a normalized conductance and susceptance spectrum that is in excellent agreement with the expected values $(\sigma=\gamma=0)$ for a rigid wall (for both the inlet and exhaust configurations). It is worth noting that the SFM method was able to educe the conductance and susceptance of the rigid wall to a higher level of precision than the LEE, CHE, and SMM methods. Typically, the CHE, LEE and SMM educed the admittance to five decimal digits of precision (i.e., five of the digits to the right of the decimal points were 
zero) whereas the SFM method educed the admittance to 12 decimal digits of precision. This is understandable since the SFM uses the exact analytical solution for the upper wall acoustic pressure to educe the impedance without the need for an optimizer. On the other hand, the CHE, LEE, and SMM use only a numerical solution for the upper wall acoustic pressure to educe the impedance. Further, the CHE and LEE methods carry the additional burden of requiring an optimizer (i.e., SDFP) to educe the impedance. When the soft wall impedance was educed, each of the four eduction methods educed the impedance spectrum given in Table 1 to the same level of precision as in the Table (i.e, three decimal digits of precision) for each flow Mach number. However, it can be assumed that if the impedance was desired to a greater level of precision than given in Table 1 (e.g., five decimal digits of precision) that the SFM would be more accurate because the exact solution is used in this method and SDFP is not required. Thus, this section confirms that all of the impedance eduction methods work flawlessly at all Mach numbers when the data is free of measurement uncertainties and the basic assumptions of the models (uniform mean flow, uniform impedance liner, etc.) are satisfied. The purpose of the following section is to test these impedance eduction models using measured data with measurement uncertainties and realistic boundary layer effects.

\section{B. Measured Data Using the Uniform Flow Assumption}

Impedance spectra are also educed using measured data acquired for five test liners. For this initial set of comparisons, the uniform mean flow assumption is used in the impedance eduction models. Each test liner is $51 \mathrm{~mm}$ wide and is tested in the GFIT using both the inlet and exhaust configurations. First, tests were conducted at a level of $130 \mathrm{~dB}$ with an upstream source (exhaust mode), as depicted in Fig. 1. These tests were conducted with a tonal source (one frequency at a time), at frequencies of 0.4 to $3.0 \mathrm{kHz}$ in $0.2 \mathrm{kHz}$ increments. The second configuration used the same test condition, with the source moved downstream (inlet mode). Each of the five test liners was chosen to have a different geometry and composition. The composition of each liner as well as comparative results are described in the following subsections.

\section{Rigid-Wall Insert}

The first test liner is a $406 \mathrm{~mm}$ long rigid-wall insert whose structure was composed of a $12.7 \mathrm{~mm}$ thick aluminum plate. The rigid-wall insert is chosen because it provides a liner for which the impedance is known a priori. It is noted that for the rigid-wall insert, the resistance and reactance values are infinite. Thus, the rigid-wall insert results are presented in terms of the normalized admittance, $\beta=1 / \zeta$. Here, the admittance is decomposed into its real and imaginary parts, $\beta=\sigma+i \gamma$, where $\sigma$ and $\gamma$ are the normalized conductance and susceptance, respectively, of the rigidwall insert. The educed values of these parameters are expected to be approximately zero for the $12.7 \mathrm{~mm}$ thick sample of aluminum.

Figures 3-5 show the educed admittance using the measured GFIT data for the rigid-wall insert for Mach 0.0, 0.3, and 0.5 , respectively. Because the SMM and SFM educed impedance could not be distinguished from each other on these graphs, it was decided to combine these results into a single graph that is denoted as SMM/SFM in the figures. At Mach 0.0 (Fig. 3), all four methods (LEE, CHE, SMM, and SFM) educed the correct admittance for both the upstream (exhaust configuration) and downstream source (inlet configuration). For the upstream source at Mach 0.3 (Fig. 4), there is generally very good agreement in educed admittance between the four impedance eduction methodologies with two exceptions. First, note that at Mach 0.3 there is some scatter observed for the upstream source near the cut on frequency (i.e., $2.7 \mathrm{kHz}$ ) of the next higher order mode (this was expected because the next higher order mode is not included in the impedance eduction analyses). Second, it was observed that SDFP returned an error code at $1.4 \mathrm{kHz}$, suggesting that the initial starting value of admittance be changed from, $\beta=0.5-0.5 i$, because of the flatness of the objective function at this frequency. Thus, SDFP has probably not converged at $1.4 \mathrm{kHz}$ (for the upstream source) at Mach 0.3 and this explains the error in educed admittance at $1.4 \mathrm{kHz}$ (see Fig. 4). Due to time constraints, the initial starting value of the normalized admittance was not changed to obtain a converged educed admittance. Note also that both the CHE and LEE are in surprisingly good agreement for the upstream source at this Mach number. Also at this Mach number there is some scatter in the educed admittance at the high frequency end of the spectrum. The accuracy of the educed admittance at Mach 0.5 (Fig. 5) is generally poor especially for the downstream source. The loss of accuracy in educed admittance at the higher Mach number was expected. This loss of accuracy may be attributed to the fact that for a duct with small cross-sectional dimensions (such as the GFIT), gradients in the mean boundary layer become more important at the higher Mach number. Although the effects of these gradients are captured in the measurement, they have been neglected in the impedance eduction models. Further, previous studies have shown ${ }^{17}$ that the effects of flow gradients in the mean boundary layer are more significant for a downstream source. This could 
explain the significant loss in accuracy of the educed admittance for the downstream source when compared to that for the upstream source.

\section{Ceramic Tubular Liner}

The second test liner is a $386 \mathrm{~mm}$ long ceramic tubular liner that has been studied extensively over the last 15 years. The ceramic tubular liner (see Fig. 6) consists of parallel, cylindrical channels embedded in a ceramic matrix. These channels, with diameter of $0.6 \mathrm{~mm}$, are perpendicular to the exposed surface and provide a surface porosity of $57 \%$. The $85.6 \mathrm{~mm}$-deep channels are rigidly terminated such that each is isolated from its neighbor to ensure a locallyreacting structure. The channel diameter is small enough that grazing flow effects are insignificant relative to internal viscous losses. The resistance of the ceramic liner is due primarily to the scrubbing losses generated as the sound propagates down the long but narrow circular channel in the ceramic matrix. This liner is chosen because it provides an impedance spectrum that varies over a range typically observed in aircraft engine nacelle liners and its resistance is expected to be independent of the mean flow Mach number.

Figure 7 shows the educed normalized impedance (i.e., the normalized resistance and normalized reactance) for the ceramic liner at Mach 0.0. All four methods are in excellent agreement for the upstream and downstream source. There is some scatter in the comparisons at the lowest frequency $(0.4 \mathrm{kHz})$ and near the anti-resonant frequency $(2.0$ $\mathrm{kHz}$ ). These are the frequencies of lowest attenuation and scatter at these two frequencies was expected because the objective function is extremely flat. Mach 0.3 results are given in Fig. 8. All four methods track the same impedance reasonably well. At this Mach number, there is some scatter in the results at the lowest frequency $(0.4 \mathrm{kHz})$ and in the vicinity of the anti-resonant frequency $(2.2 \mathrm{kHz})$, and also near the high frequency end of the spectrum. The scatter at 0.4 and $2.2 \mathrm{kHz}$ is due to the low attenuation but the scatter at the high frequency end of the spectrum results from the cut on of higher order duct modes (these high-order duct moder are neglected in the impedance eduction models). Note that for each impedance eduction method, there is an insignificant difference in the impedance educed at Mach 0.0 and that educed at Mach 0.3. This confirms that the impedance of the ceramic tubular liner is nearly independent of the flow Mach number. Figure 9 shows the educed normalized impedance at the highest Mach number (Mach 0.5). There is unacceptable scatter in educed impedance among the impedance eduction methodologies. This scatter is greater for the downstream source and indications are that it may be due to neglecting the mean flow boundary layer in the impedance eduction methods.

\section{Wire Mesh Liner}

The third test liner is a $610 \mathrm{~mm}$ long wire mesh liner (see Fig. 6). The channels have a hexagonal shape, with a nominal diameter of approximately $10 \mathrm{~mm}$ and a depth of $76 \mathrm{~mm}$. The resistance of the wire mesh liner is $270 \mathrm{MKS}$ Rayls and this resistance is due primarily to the wire mesh facesheet. This liner is chosen because its resistance is expected to be independent of the source sound pressure level and flow Mach number. Figure 10 shows the educed normalized impedance for the wire mesh liner at Mach 0.0. All four methods are in excellent agreement except near the anti-resonant frequency $(2.4 \mathrm{kHz})$ where the measured attenuation was determined to be too low to get an accurate impedance eduction. Results for Mach 0.3 are given in Fig. 11. For the upstream source all four methods track the same impedance extremely well, although as expected there is some scatter in the results near the anti-resonant frequency $(2.4 \mathrm{kHz})$. There is more scatter among the impedance eduction methods for the downstream source especially at the lower frequencies where the attenuation is low. Finally, Fig. 12 shows the wire mesh liner impedance eductions at the highest Mach number (Mach 0.5). Although the upstream source results compare well (especially the normalized reactance), there is unacceptable scatter in educed impedance for the downstream source.

\section{Conventional Liners}

The fourth and fifth test liners are conventional, perforate-over-honeycomb liners (see Fig. 6) that are representative of liners commonly used in current aircraft engine nacelles. The fourth liner has a facesheet with $0.99 \mathrm{~mm}$-diameter holes and a thickness of $0.635 \mathrm{~mm}$, which is bonded to a $38.18 \mathrm{~mm}$-deep core consisting of hexagonal shaped channels with a nominal diameter of $10 \mathrm{~mm}$, and is terminated with a rigid back plate. The surface porosity is $8.7 \%$. The fifth test liner is identical to the fourth, but with a porosity of $13.2 \%$. These liners are chosen because their resistance spectrum is expected to be nonlinear with respect to the mean flow Mach number. That is, as the flow Mach number is increased, their resistance is expected to increase. Further, the high porosity conventional liner is expected to provide a lower resistance than the low porosity liner. 
Impedances educed for the low porosity conventional liner at Mach 0.0 are given in Fig. 13. Except near the high frequency end of the spectrum (i.e., $3.0 \mathrm{kHz}$ ) where higher order modes are cut on, the four impedance eduction methods educe nearly identical impedances. Note that the normalized resistance has a low value and is nearly independent of frequency. The reactance follows a $-\cot (k d)$ behavior (as expected). Here $d$ is the depth of the cavity. Surprisingly, the three eduction methods track one another better for this conventional liner than for the rigid-wall insert and the ceramic tubular liner. Figure 14 shows results when the Mach number of the low porosity conventional liner is increased to Mach 0.3. The impedance eduction methods are in excellent agreement with each other except in the extreme high frequency end of the spectrum where higher order modes are cut on and at $0.4 \mathrm{kHz}$ where the attenuation is low (Fig. 14). There is a significant increase in the normalized resistance (compared to the Mach zero value), confirming that the resistance of the conventional liner is nonlinear with respect to the flow Mach number. It is observed that at Mach 0.3 the resistance is almost independent of frequency and the reactance curve again follows $\mathrm{a}-\cot (k d)$ behavior, although there is a variation from this trend at frequencies above $2.6 \mathrm{kHz}$ for the CHE and LEE cases. The bending of the reactance curve downward at the extreme high frequency end of the spectrum has been observed for other liners. It is reassuring, however, that each impedance eduction method predicts this trend.

When the Mach number of the low porosity liner is increased to Mach 0.5 (see Fig. 15) there is significant scatter in the educed normalized impedance predicted among the eduction methods. This scatter is again considerably worse for the downstream source. Note that for the upstream source at the higher Mach number, the two eduction methods that use single mode analysis (i.e., SMM and SFM) predict a reactance spectrum that follows a $-\cot (k d)$ behavior whereas the CHE and LEE continue to show a bending of the reactance curve away from this behavior. This could suggest that the bending of the reactance curve is a near field effect created at the leading/trailing edges of the liner. This near field effect cannot be captured using a single mode analysis such as that used by the SMM or the SFM. Impedances were also educed for the high porosity conventional liner and although the educed resistance was lower (as expected), the observed trends among the impedance eduction techniques were the same as for the low porosity conventional liner. Consequently, the high porosity conventional liner results have not been presented for the sake of brevity.

\section{Shear Flow Effects}

Detailed mean flow profile measurements were taken for the wire mesh liner. These profile measurements were taken at various locations across the span of the GFIT with the wire mesh liner installed for both the inlet and exhaust configuration. The results reported here use the mean flow profile taken at the duct's midspan (i.e., $y=W / 2$ ). The two analyses that account for the effects of the 1D shear (i.e., the LEE and SMM) are run to evaluate the effects of the shear flow. It is expected that the effects of the shear layer on the impedance of the wire mesh liner should be minimal.

Figure 16 compares the educed impedance at Mach 0.3 with the measured flow profile inserted into the LEE and SMM. These results are labeled LEE (Shear) and SMM (Shear) in the figure. The impedance educed using the LEE with uniform flow (i.e., LEE (Uniform)) is used as the baseline for comparison purposes. The LEE code with shear and the SMM tracks the impedance educed using the uniform flow LEE code well except near the antiresonant frequency (where the attenuation is low) and near the high frequency end of the spectrum (where higher order modes are cut on). There is more scatter (among the methodologies) in the downstream source eductions than for the upstream source. Figure 17 shows results at the higher Mach number (Mach 0.5). For the upstream source, the educed resistance from the three methods track one another well except near the antiresonant frequency (i.e., $2.4 \mathrm{kHz}$ ) and the high frequency end of the spectrum where high-order modes are cuton. However, the educed reactance of the upstream source at this higher Mach number do not track each other well at frequencies above $1.5 \mathrm{kHz}$. Further, the educed reactance spectrum is flatter when compared to that at Mach 0.3. Note that at this Mach number (Mach 0.5) there is unacceptable scatter in the educed impedance (among the three methods) for the downstream source. It is worth noting that the relatively small change in educed impedance of the LEE (Shear) and SMM (Shear) compared to LEE (Uniform) for Mach 0.3 was expected for the wire mesh liner. A much larger effect of the shear flow is expected for a liner that is more nonlinear with respect to the flow Mach number, such as a conventional liner. However, detail flow profile measurement were not acquired for the conventional liner and the effects of the shear flow on the conventional liner could not be investigated. This investigation will be conducted at a later date.

\section{Conclusions}

In this paper a comparative study of four commonly used impedance eduction techniques is performed. The comparative study is performed using identical test conditions and on a database of five test liners. A preliminary 
assessment of the effects of the shear flow on the wire mesh liner is also investigated. Based on the results of this study, the following conclusions are drawn:

1. For uniform flow Mach numbers of 0.3 or less the impedance eduction techniques educe nearly identical normalized impedances except at frequencies in the spectra where the liner attenuation is low. These frequencies are generally at the extreme low frequency end of the impedance spectrum and near the anti-resonant frequencies.

2. For a Mach number of 0.5 , there is considerable scatter in the impedance educed from the uniform flow eduction methods (especially for the downstream source). This scatter appears due to the absence of some essential physics in the eduction models. Indications are that the absence of the mean boundary layer is a reason for this scatter.

3. There is generally better agreement (in educed impedance) among the eduction models for the conventional liner than for the rigid-wall insert, the ceramic, or the wire mesh liner.

4. For the conventional liner, there is a tendency for the educed reactance curve to bend downward away from the expected $-\cot (k d)$ behavior at high Mach numbers and at the high frequency end of the educed impedance spectrum. This trend is reproduced by all of the eductions methods and warrants further investigation.

5. The effect of the mean boundary layer has little effect on the educed impedance of the wire mesh liner at Mach 0.3 . When the Mach number is increased to 0.5, the slope of the reactance spectrum is slightly decreased when an upstream source is used, but the results for a downstream source experience increased scatter. Further investigation of these shear flow effects will be the focus of future investigations.

\section{Acknowledgement}

This work was funded by NASA's Fixed Wing Project of the Fundamental Aeronautics Program.

\section{References}

\footnotetext{
${ }^{1}$ Armstrong, D. L., Beckemeyer, R. J., and Olsen, R. F., "Impedance Measurements of Acoustic Duct Liners With Grazing Flow," Paper presented at the 87th Meeting of the Acoustical Society of America, New York, NY, 1974.

${ }^{2}$ Watson, W. R., Tanner, S. E.., Jones, M. G., and Parrott, T. L., "Optimization Method for Educing Variable-Impedance Liner Properties," AIAA Paper 97-1704, June, 1997.

${ }^{3}$ Watson, W. R., Jones, M. G., and Parrott, T. L., "Validation of an Impedance Eduction Method in Flow," AIAA Journal, Vol. 37, No. 7, July 1999, pp. 818-824.

${ }^{4}$ Jones, M. G., Watson, W. R., Tracy, M. B., and Parrott, T. L., "Comparison of Two Waveguide Methods for Educing Liner Impedance in Grazing Flow," AIAA Journal, Vol. 42, No. 2, 2004, pp. 232-240.

${ }^{5}$ Jones, M. G., Parrott, T. L., and Watson, W. R., "Comparison of Acoustic Impedance Eduction Techniques for Locally-Reacting Liners," AIAA Paper 2003-3306, May 2003.

${ }^{6}$ Syed, A. A., "The Steady Flow Resistance of Perforated Sheet Materials in High Speed Grazing Flows,” NASA CR-2002-211749, 2002.

${ }^{7}$ Gallman, J. M. and Kunze, R. K., "Grazing Flow Acoustic Impedance Testing for the NASA AST Program,” AIAA Paper 2002-2447, June 2002.

${ }^{8}$ Watson, W. R., Jones, M. G., and Parrott, T. L., "Comparison of a Convected Helmholtz and Euler Model for Impedance Eduction in Flow," AIAA Paper 2006-2643, May 2006.

${ }^{9}$ Elnady, T. M., Boden, H., and Elhadidi, B., "Validation of an Inverse Semi-Analytical Technique to Educe Liner Impedance," AIAA Journal, Vol. 47, No. 12, December 2009, pp. 2836-2844.

${ }^{10}$ Jing, X., Peng, S., and Sun, X., “A Straightforward Method for Wall Impedance Eduction in a Flow Duct,” J. Acoust. Soc. Am., Vol. 124, No. 1, July 2008.

${ }^{11}$ Myers, M. K., "On the Acoustic Boundary Condition in the Presence of Flow," Journal of Sound and Vibration, Vol. 71, No. 3, 1980, pp. $429-434$.

${ }^{12}$ Kraft, R. E., “Theory and Measurements of Acoustic Wave Propagation In Multi-Segmented Rectangular Ducts,” P.h.D. Thesis, University of Cincinnati, 1976.

${ }^{13}$ Stewart, G. W., III, “A Modification of Davidon's Minimization Method to Accept Difference Approximations of Derivatives," Journal of ACM, Vol. 14, No. 1, 1967, pp 72-83.

${ }^{14}$ Pridmore-Brown, D. C., "Sound propagation in a fluid flowing through an attenuating duct," Journal of Fluid Mechanics, Vol. 4, 1958, pp $393-406$.

${ }^{15}$ Mungur, P. and Gladwell, G.M.L., "Acoustic Wave Propagation in a Sheared Fluid Contained in a Duct," Journal of Sound Vibration, Vol. 9 , No. 1, July 1969 , pp. $28-48$.

${ }^{16}$ Snider, R., “Attenuation Predictions for Segmented Liners in Supersonic Flow.” M.S, Thesis, The George Washington University, July 1993.

${ }^{17}$ Nayfeh, A. H., Kaiser, J. E., and Telionis, D. P. , “The Acoustics of Aircraft Engine Ducts," AIAA Journal, Vol. 11, No. 2, pp. 150-153, 1973.
} 


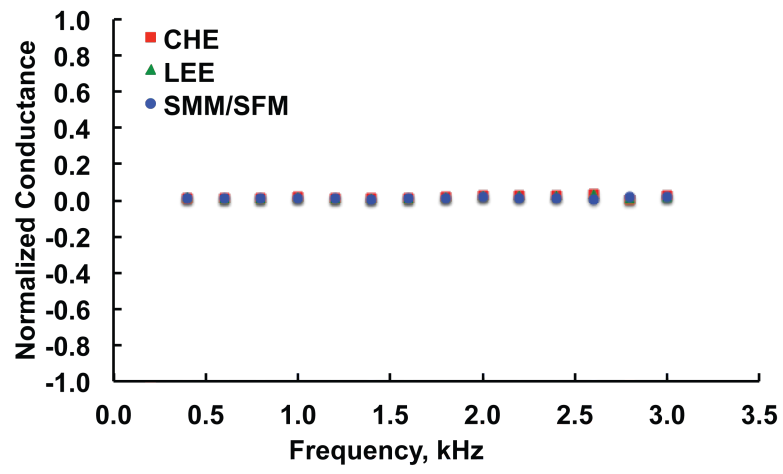

(a) Upstream source

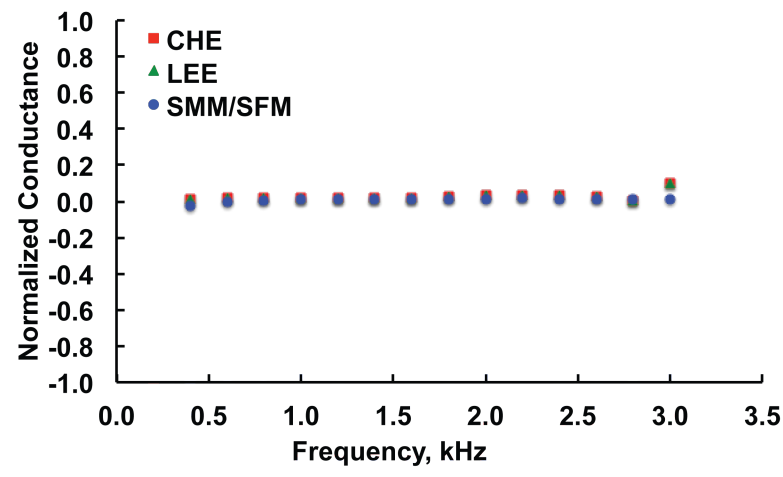

(c) Downstream source

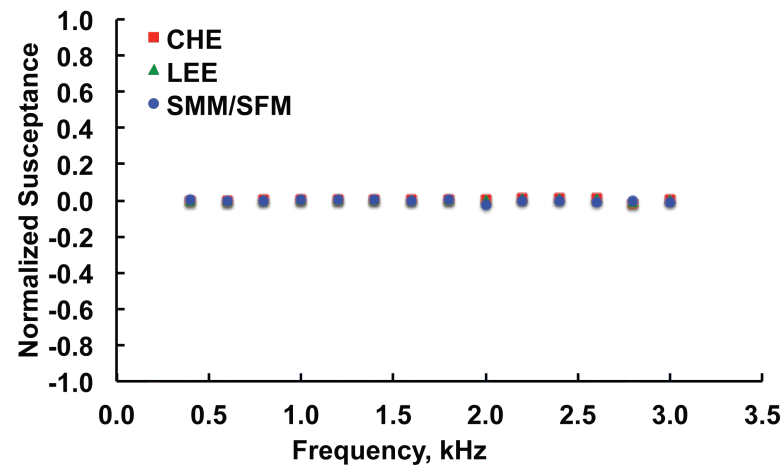

(b) Upstream source

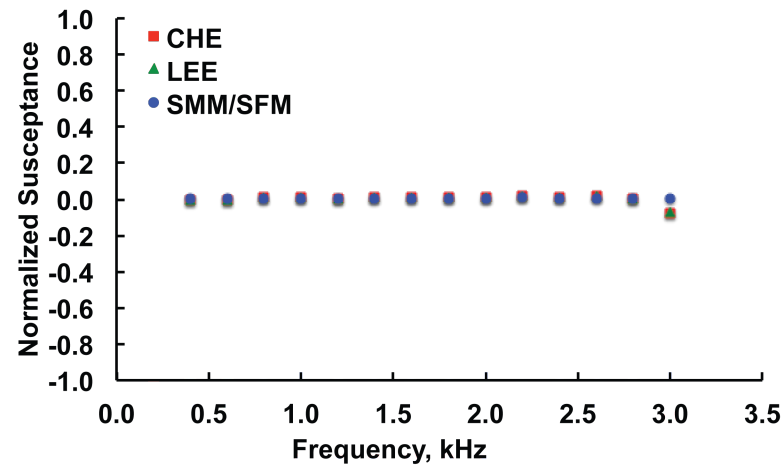

(d) Downstream source

Figure 3. Educed normalized admittance for the rigid-wall insert at Mach 0.0 using measured data from the GFIT. 


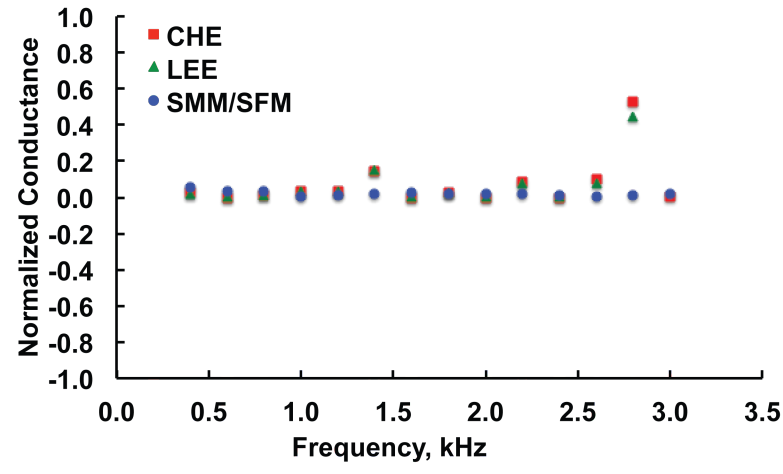

(a) Upstream source

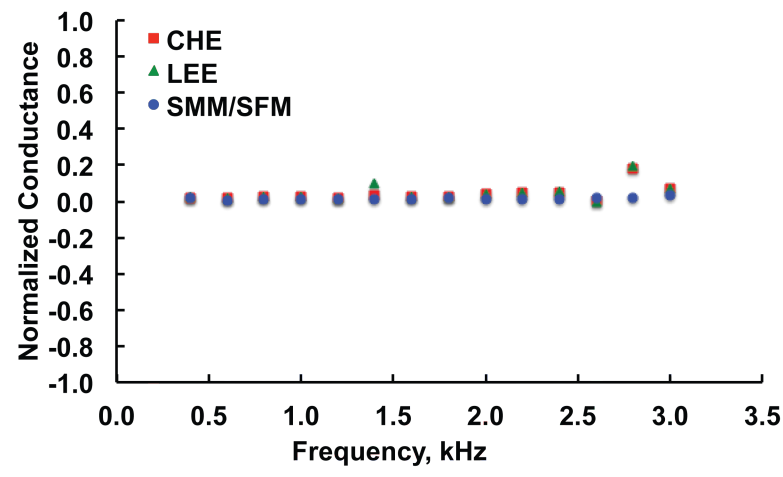

(c) Downstream source

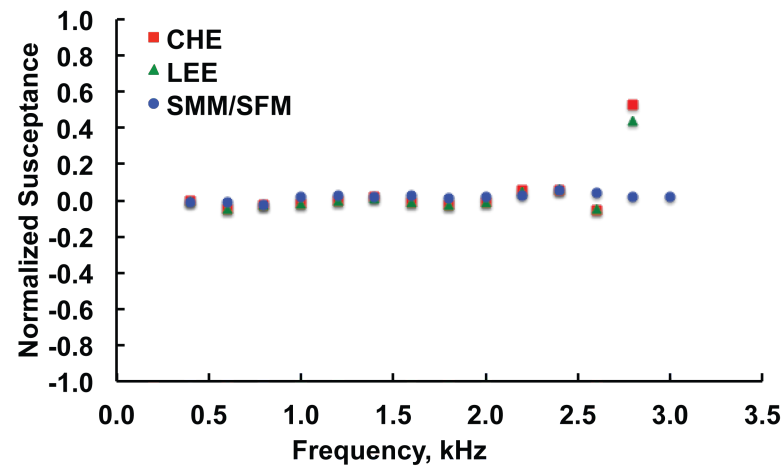

(b) Upstream source

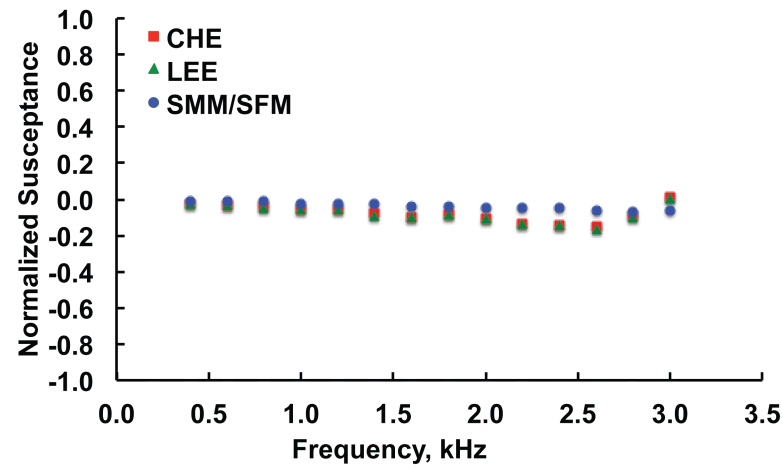

(d) Downstream source

Figure 4. Educed normalized admittance for the rigid-wall insert at Mach 0.3 using measured data from the GFIT. 


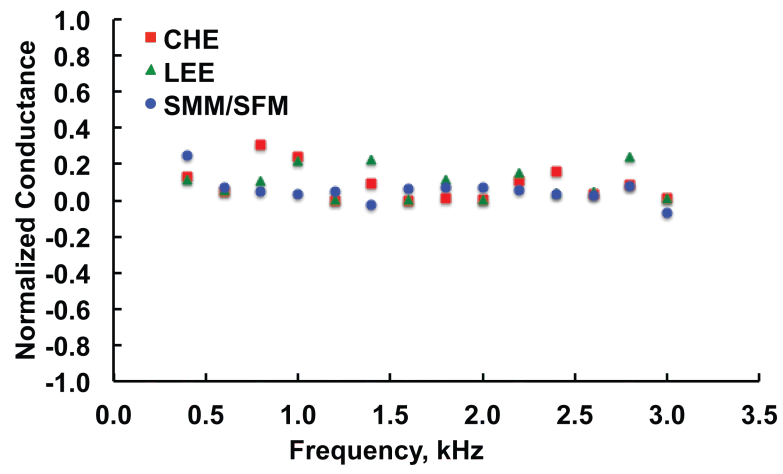

(a) Upstream source

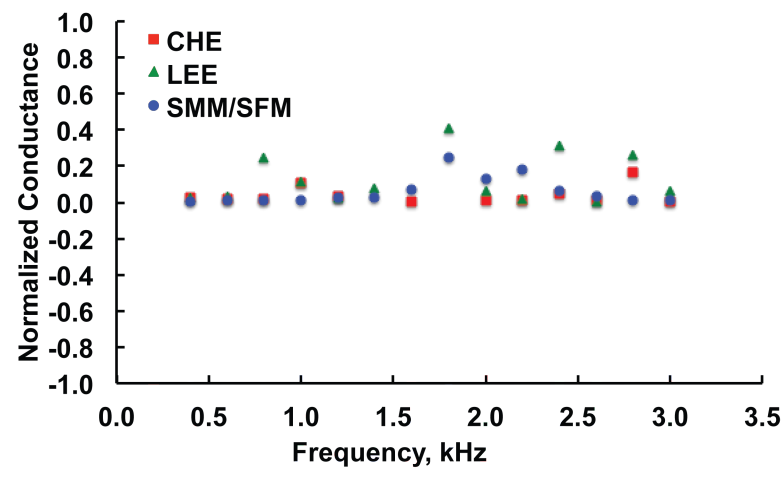

(c) Downstream source

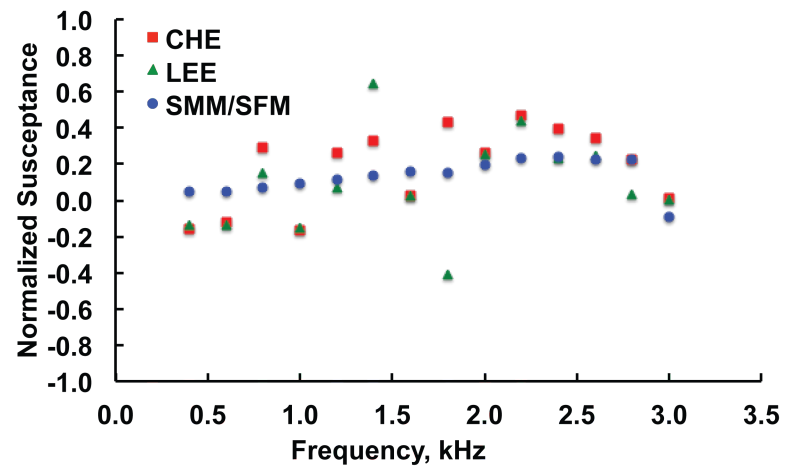

(b) Upstream source

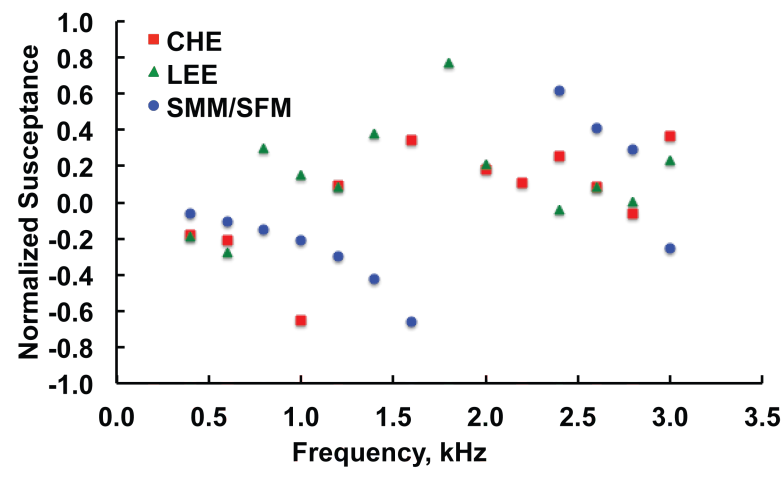

(d) Downstream source

Figure 5. Educed normalized admittance for the rigid-wall insert at Mach 0.5 using measured data from the GFIT. 


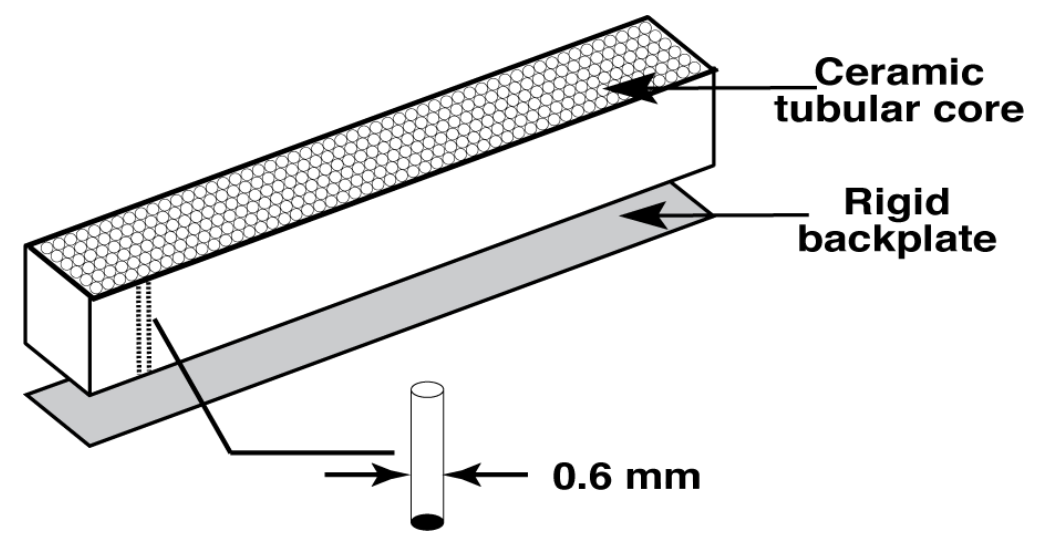

(a) Ceramic tubular liner

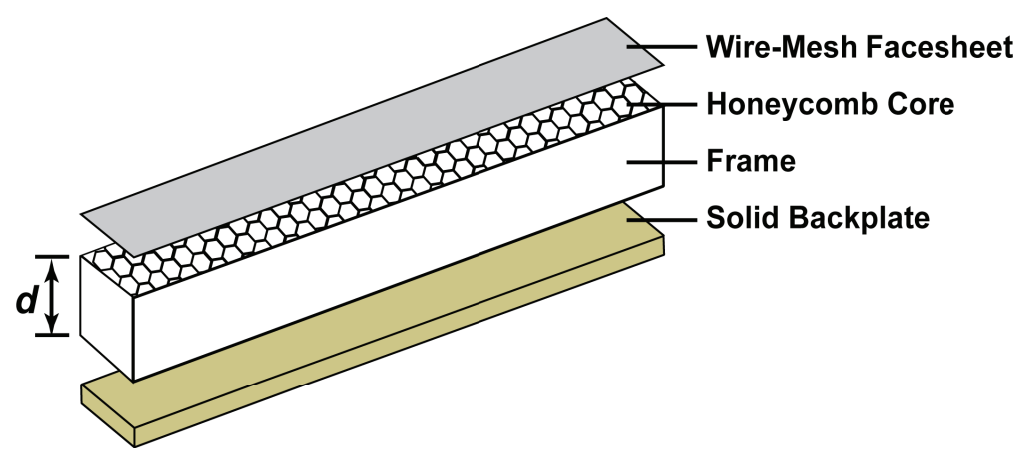

(b) Wire Mesh Liner

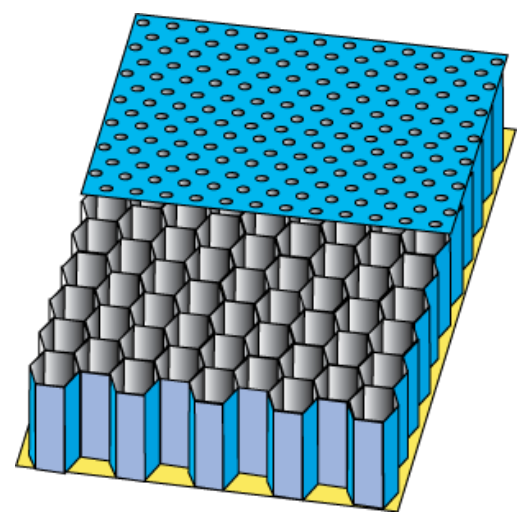

(c) Conventional liner

Figure 6. Schematic of test liners. 


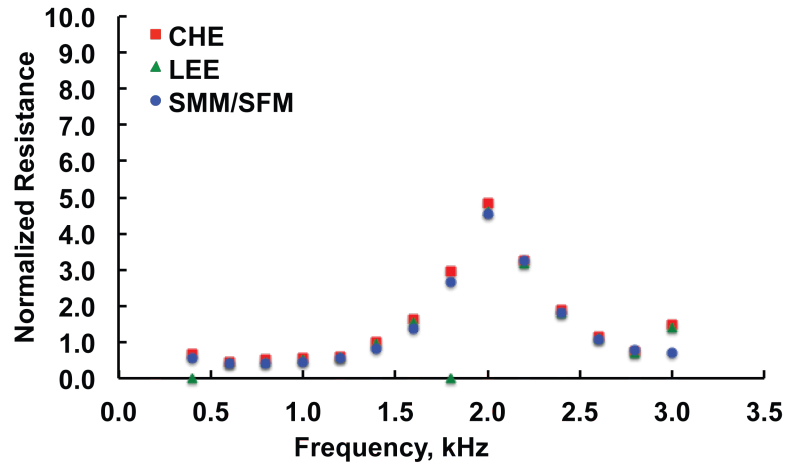

(a) Upstream source

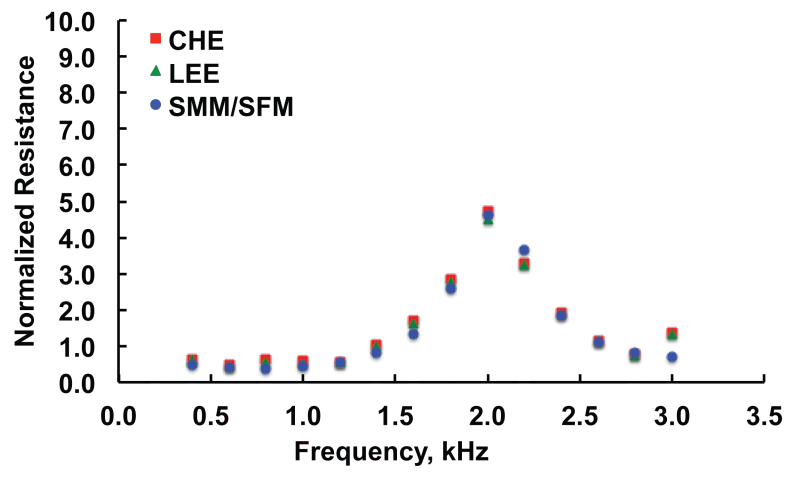

(c) Downstream source

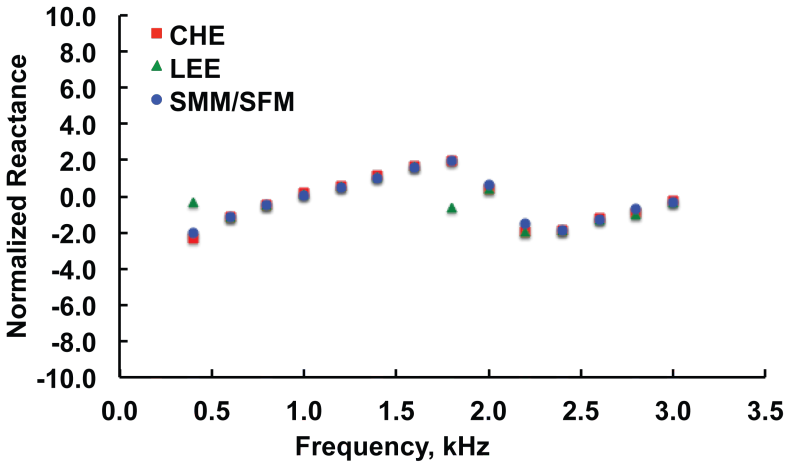

(b) Upstream source

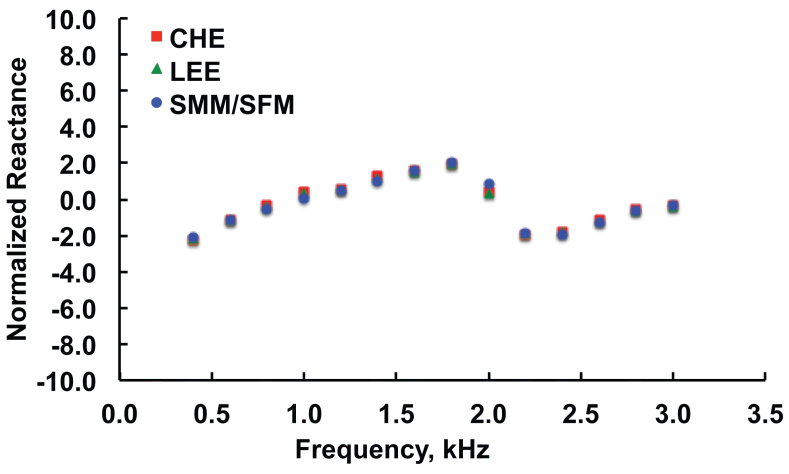

(d) Downstream source

Figure 7. Educed normalized impedance for the ceramic tubular liner at Mach 0.0 using measured data from the GFIT. 


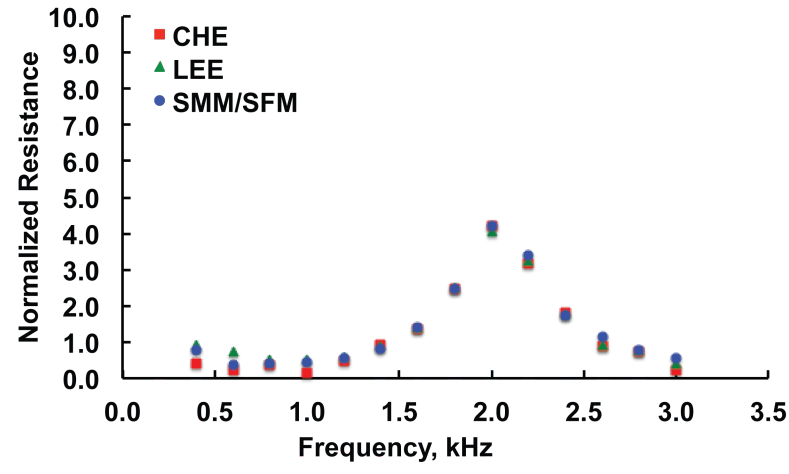

(a) Upstream source

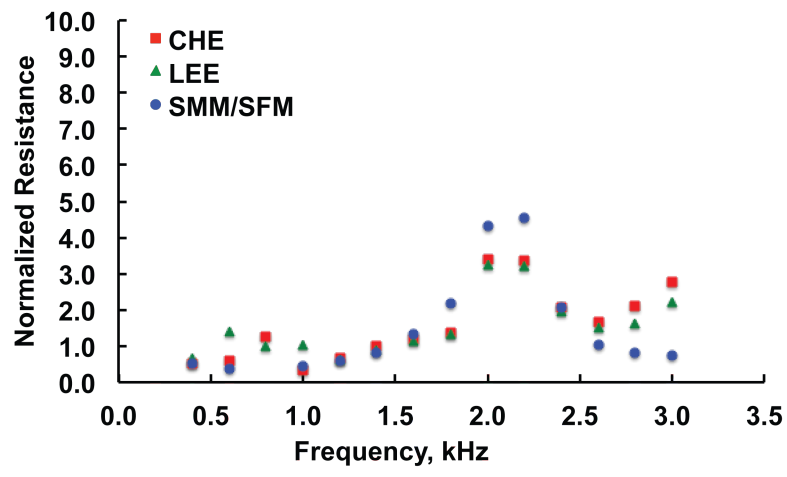

(c) Downstream source

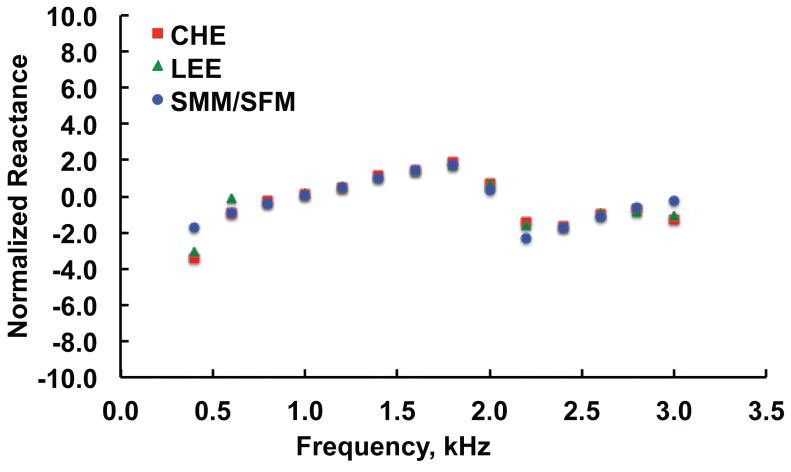

(b) Upstream source

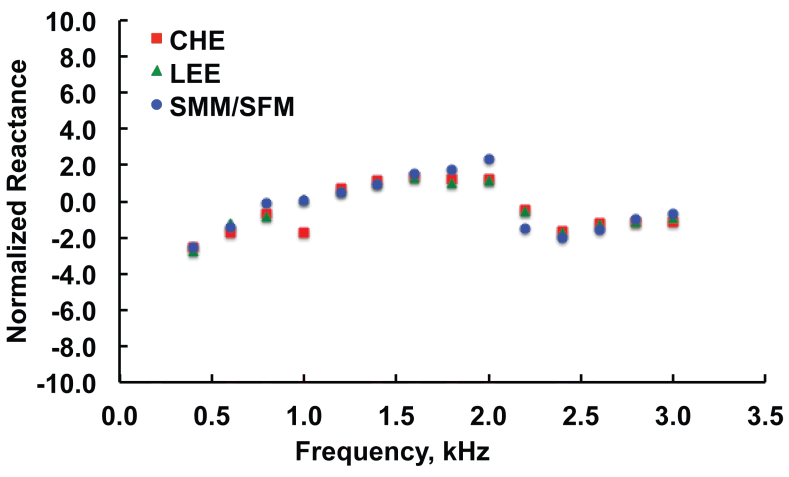

(d) Downstream source

Figure 8. Educed normalized impedance for the ceramic tubular liner at Mach 0.3 using measured data from the GFIT. 


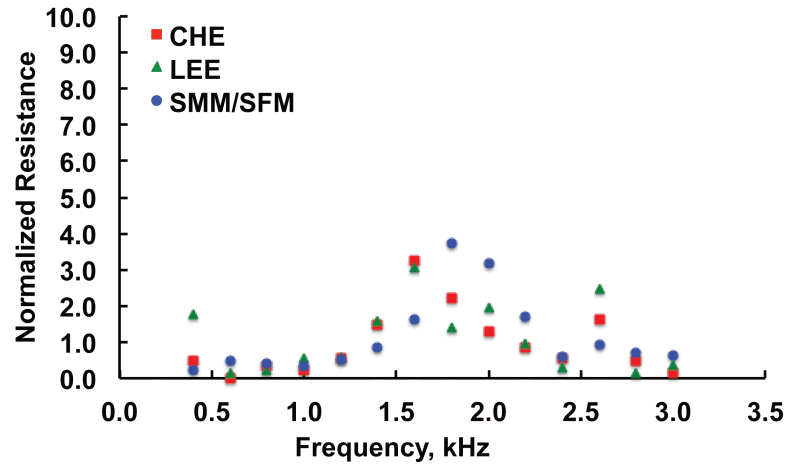

(a) Upstream source

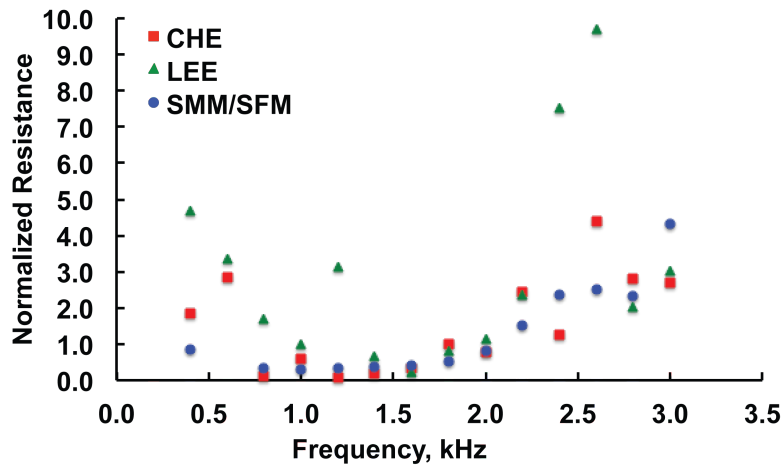

(c) Downstream source

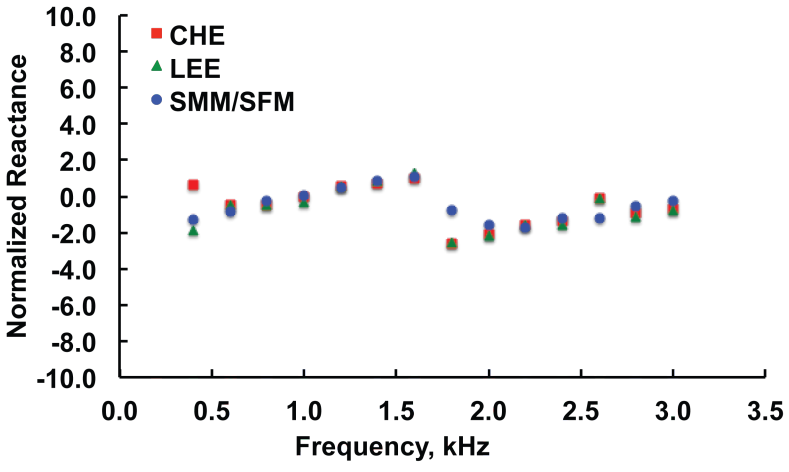

(b) Upstream source

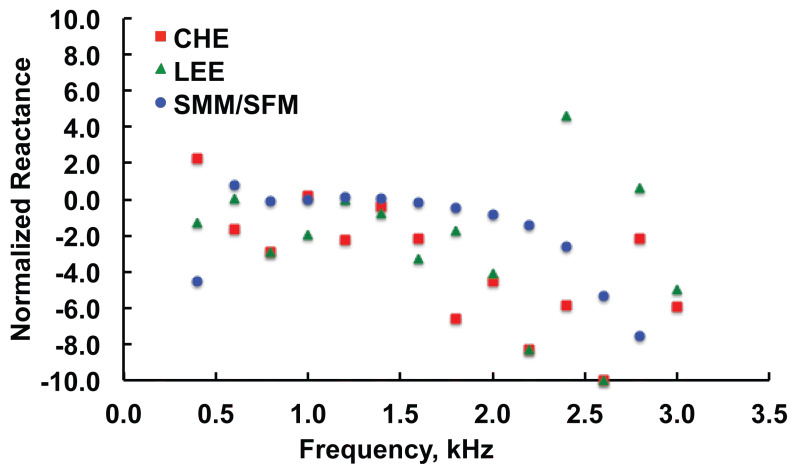

(d) Downstream source

Figure 9. Educed normalized impedance for the ceramic tubular liner at Mach 0.5 using measured data from the GFIT. 


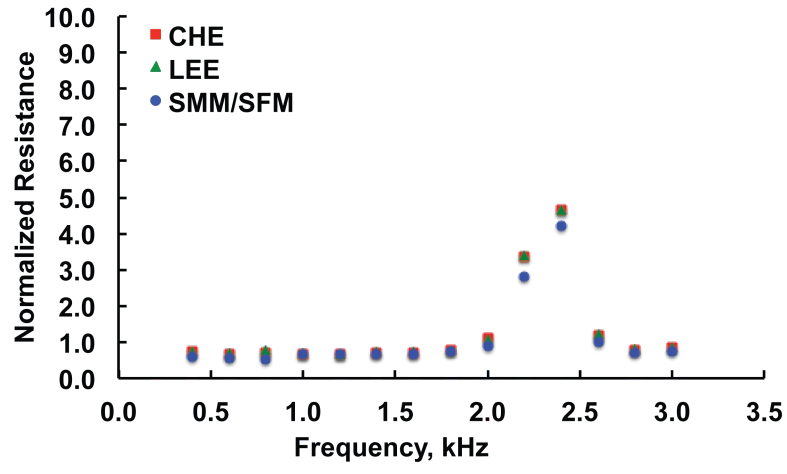

(a) Upstream source

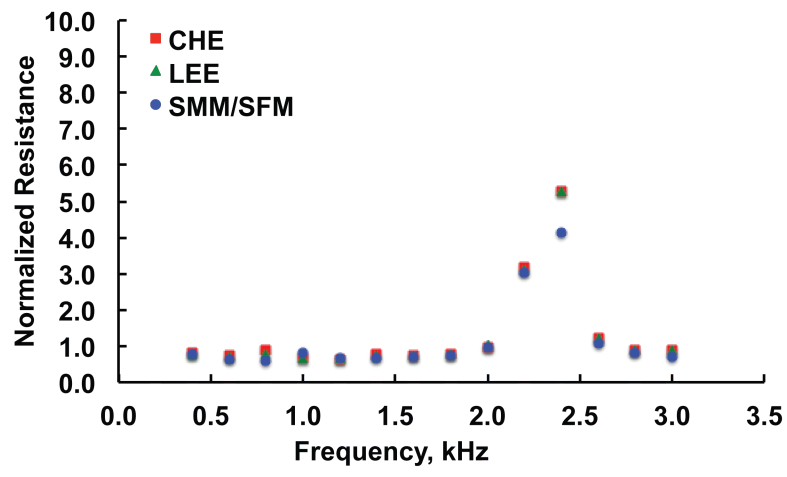

(c) Downstream source

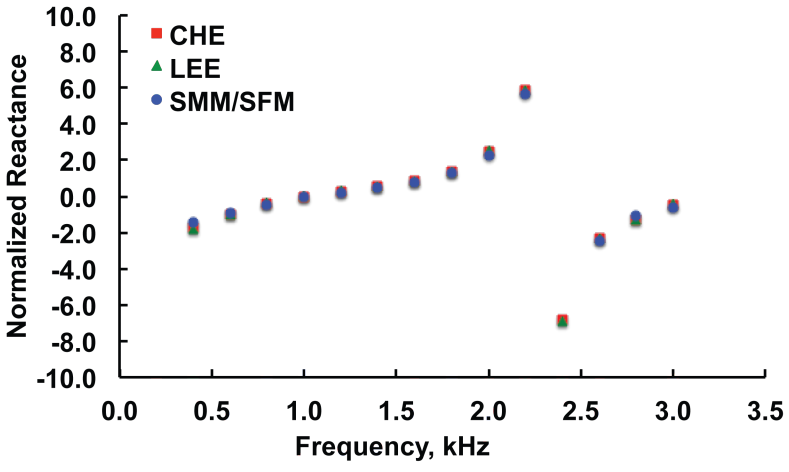

(b) Upstream source

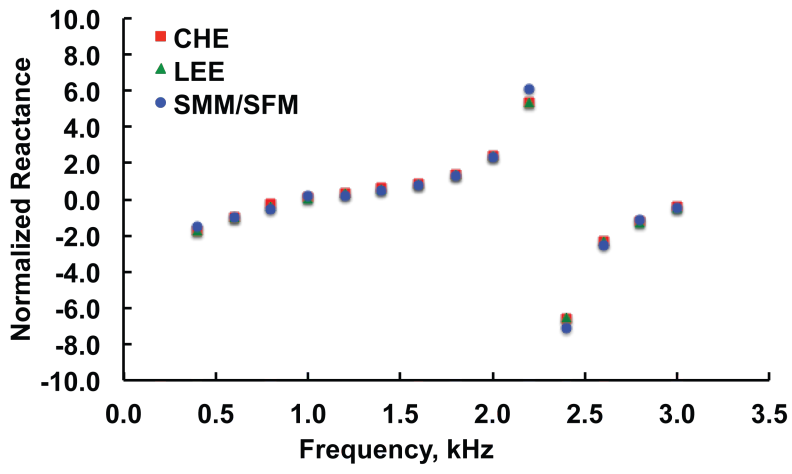

(d) Downstream source

Figure 10. Educed normalized impedance for the wire mesh liner at Mach 0.0 using measured data from the GFIT. 


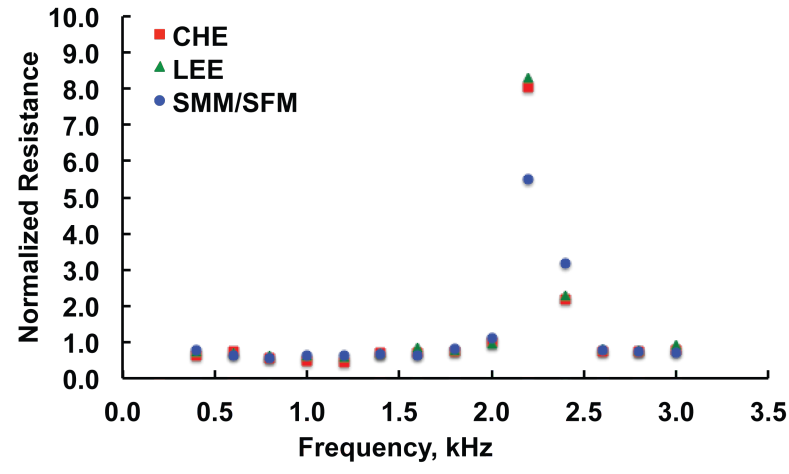

(a) Upstream source

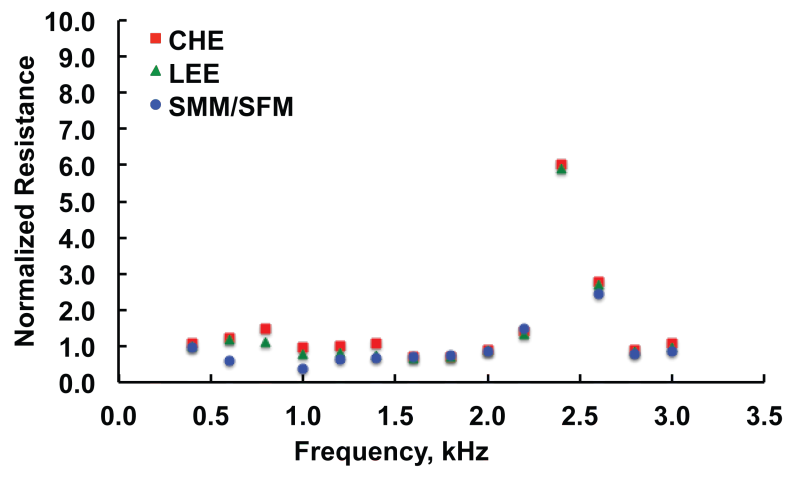

(c) Downstream source

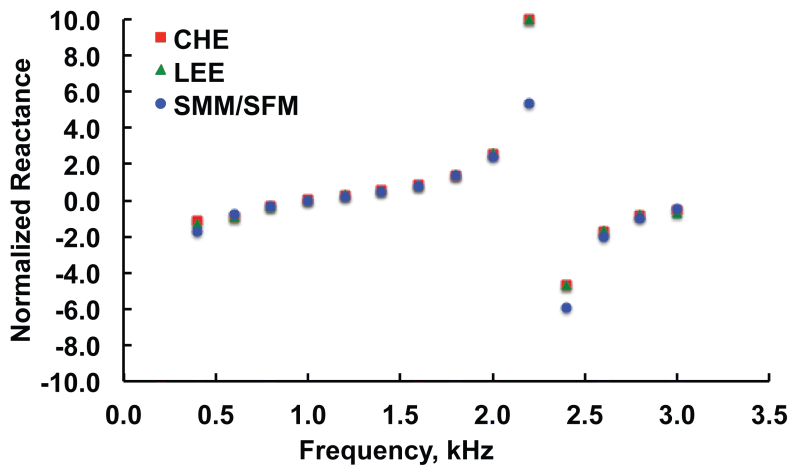

(b) Upstream source

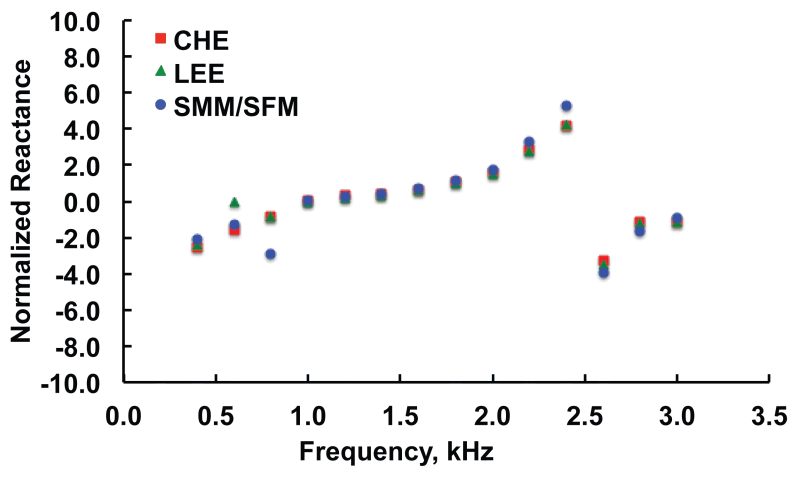

(d) Downstream source

Figure 11. Educed normalized impedance for the wire mesh liner at Mach 0.3 using measured data from the GFIT. 


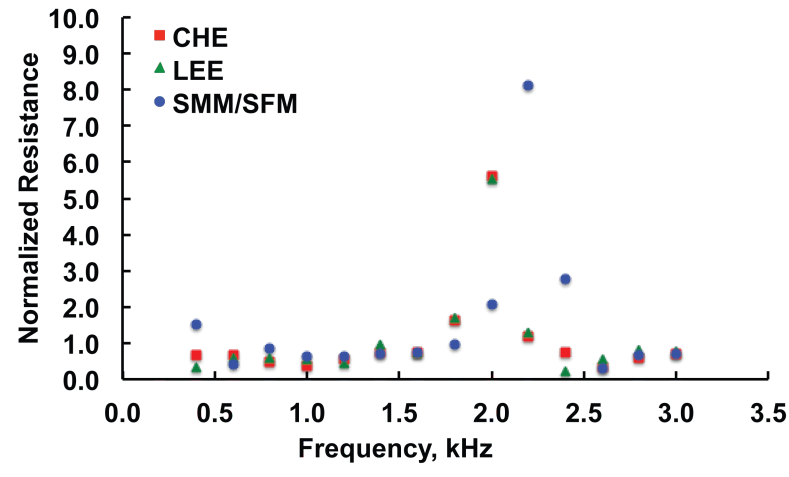

(a) Upstream source

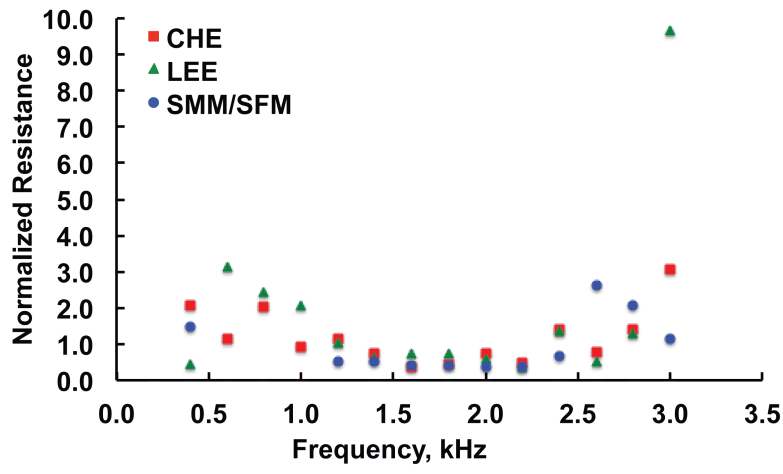

(c) Downstream source

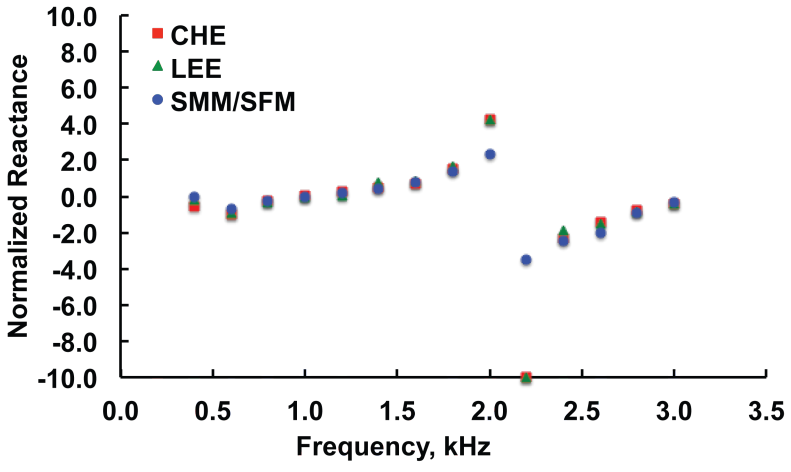

(b) Upstream source

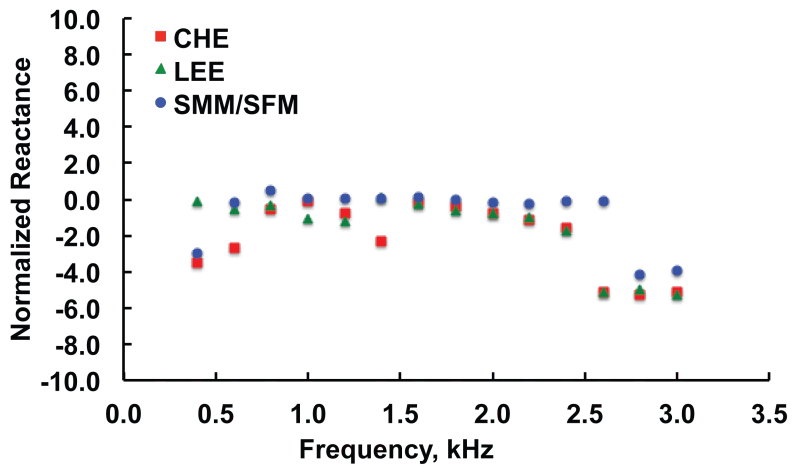

(d) Downstream source

Figure 12. Educed normalized impedance for the wire mesh liner at Mach 0.5 using measured data from the GFIT. 


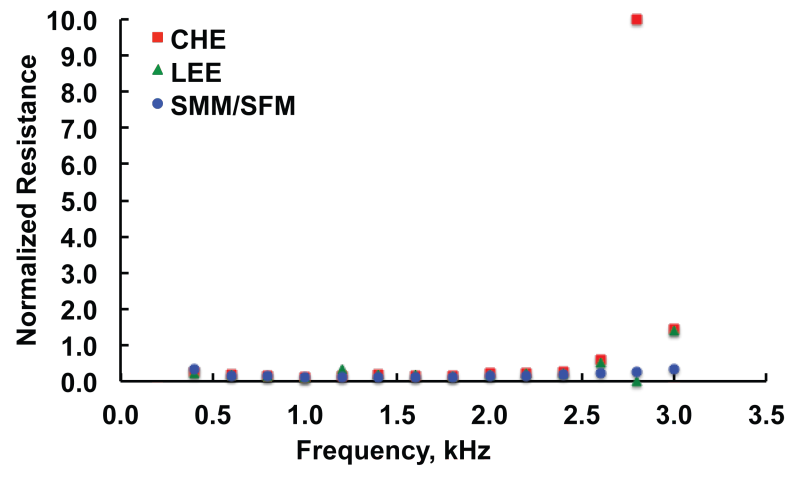

(a) Upstream source

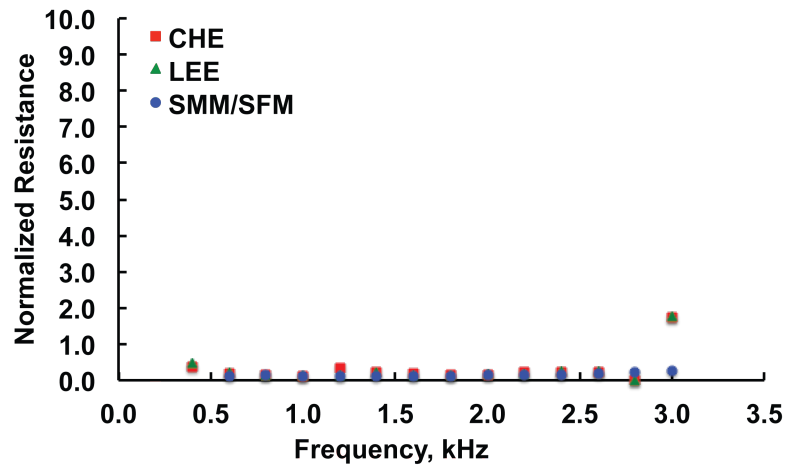

(c) Downstream source

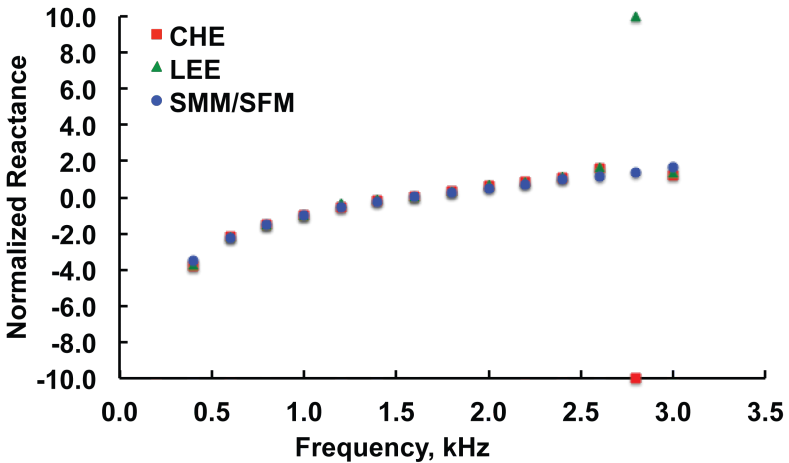

(b) Upstream source

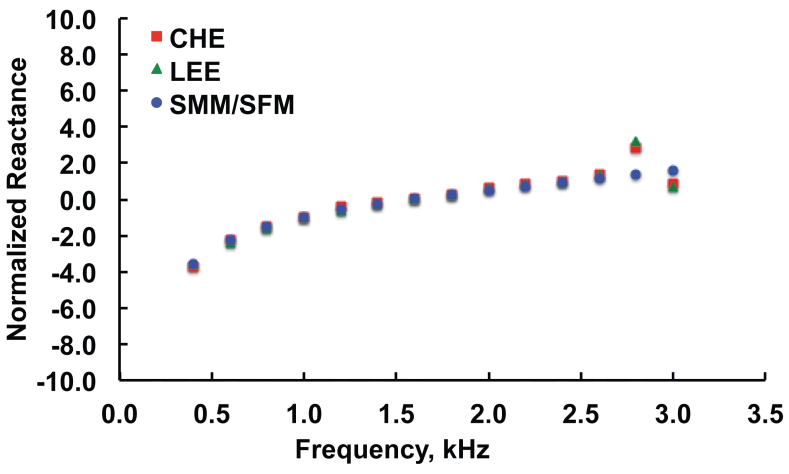

(d) Downstream source

Figure 13. Educed normalized impedance for the low porosity conventional liner at Mach 0.0 using measured data from the GFIT. 


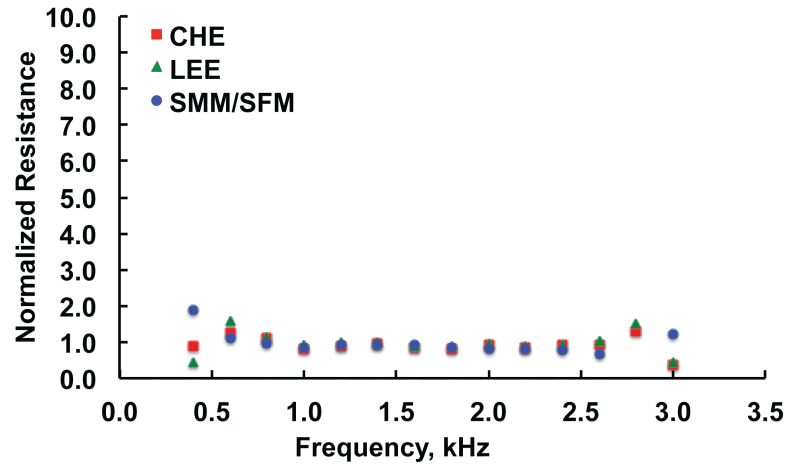

(a) Upstream source

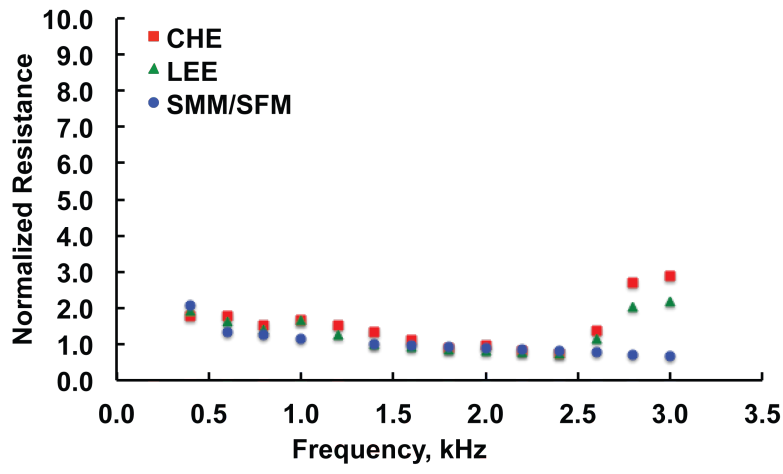

(c) Downstream source

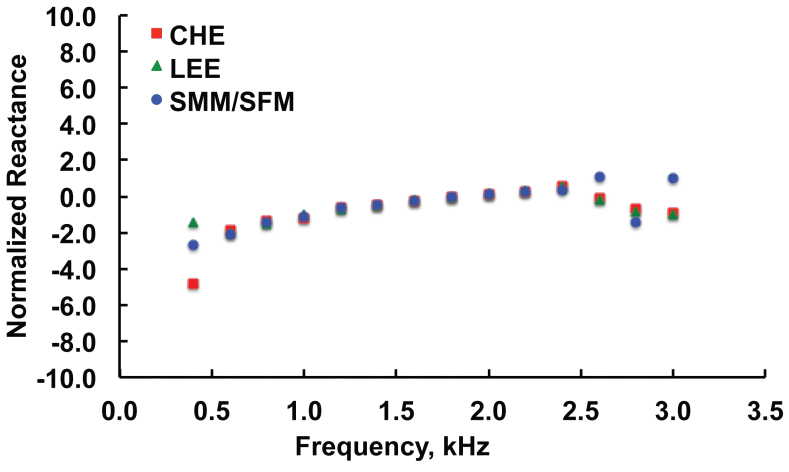

(b) Upstream source

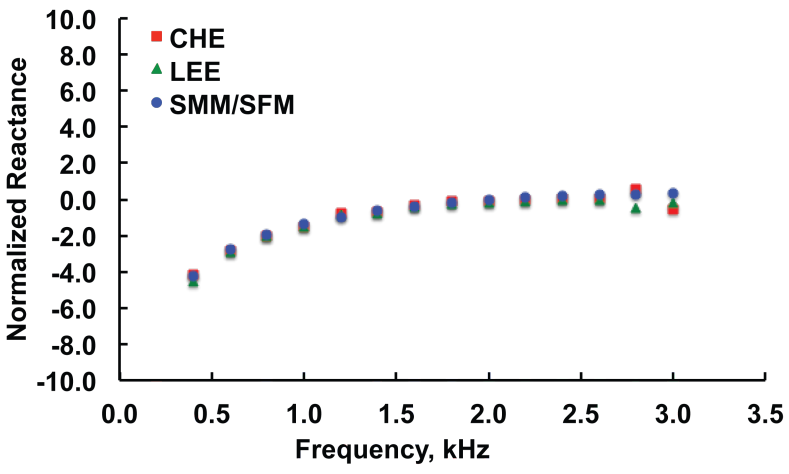

(d) Downstream source

Figure 14. Educed normalized impedance for the low porosity conventional liner at Mach 0.3 using measured data from the GFIT. 


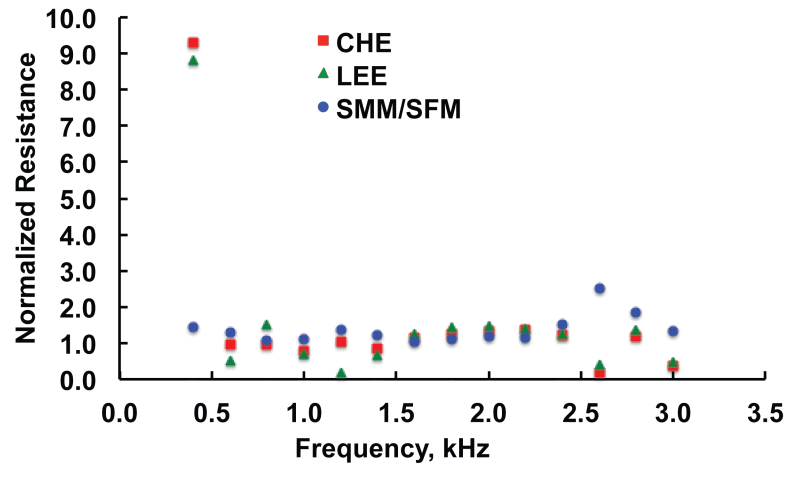

(a) Upstream source

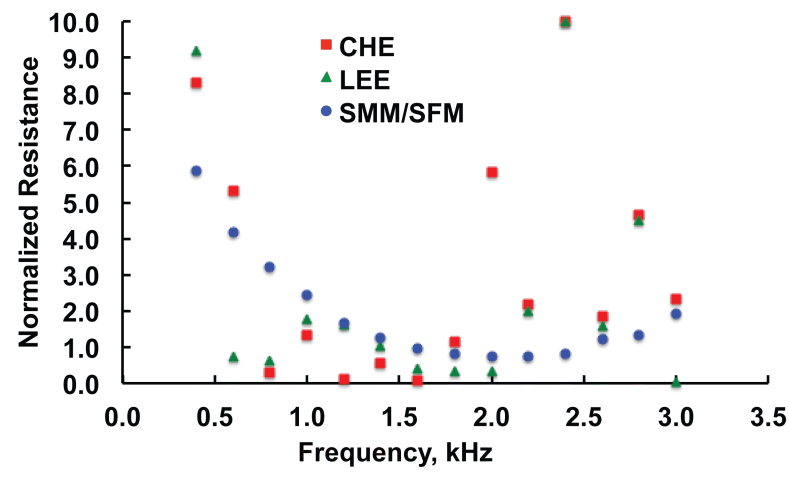

(c) Downstream source

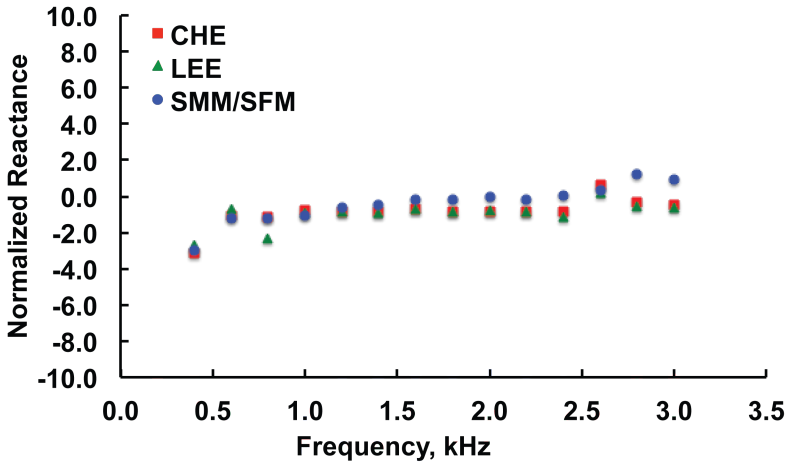

(b) Upstream source

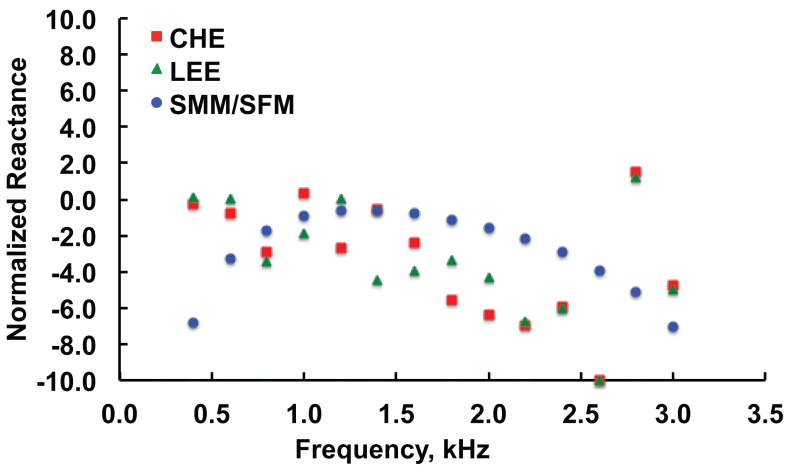

(d) Downstream source

Figure 15. Educed normalized impedance for the low porosity conventional liner at Mach 0.5 using measured data from the GFIT. 


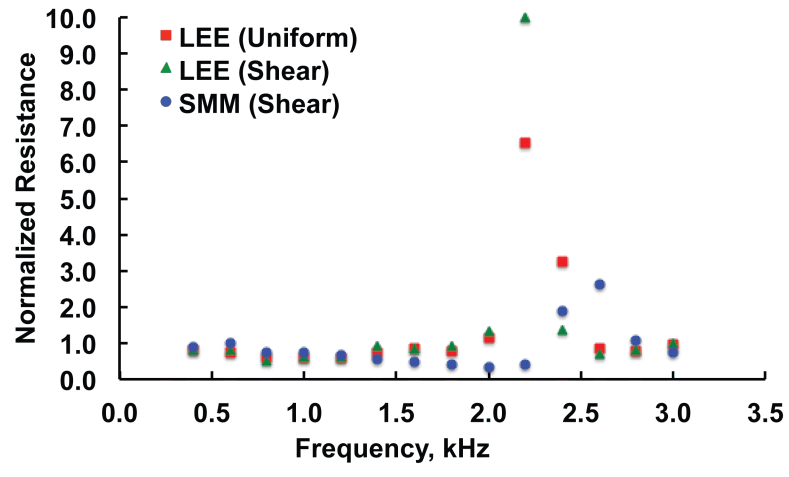

(a) Upstream source

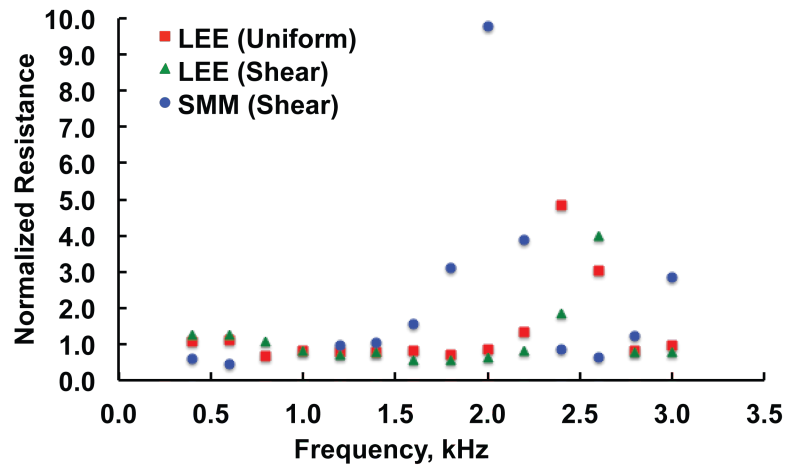

(c) Downstream source

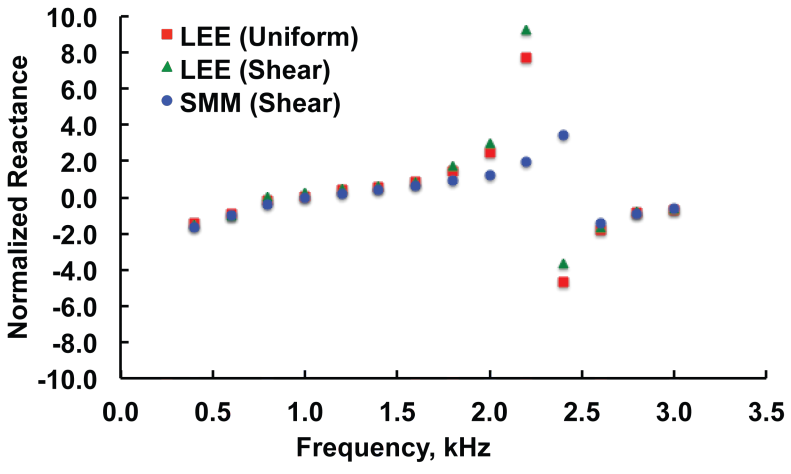

(b) Upstream source

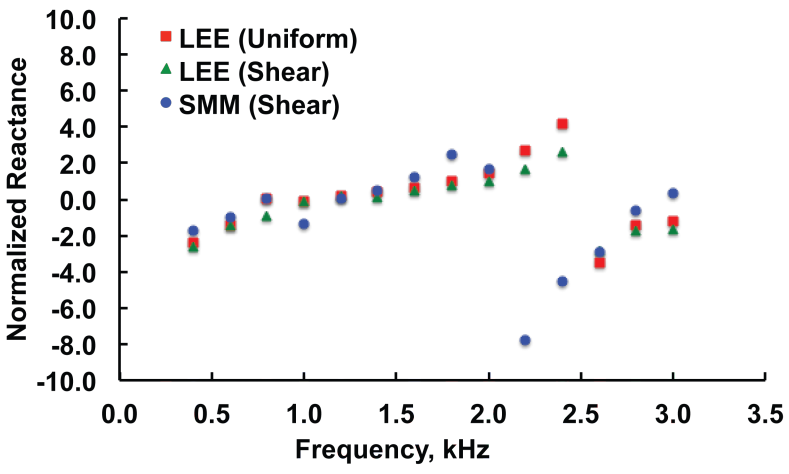

(d) Downstream source

Figure 16. Educed normalized impedance for the wire mesh liner at Mach 0.3 using measured data from the GFIT with mean shear. 


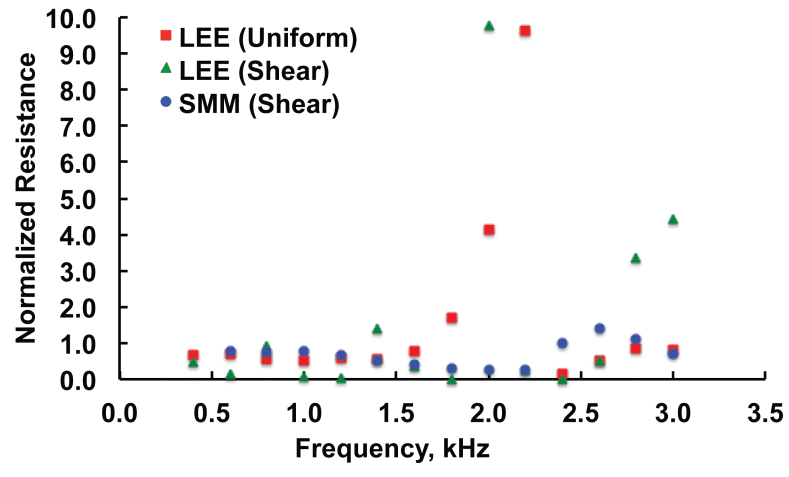

(a) Upstream source

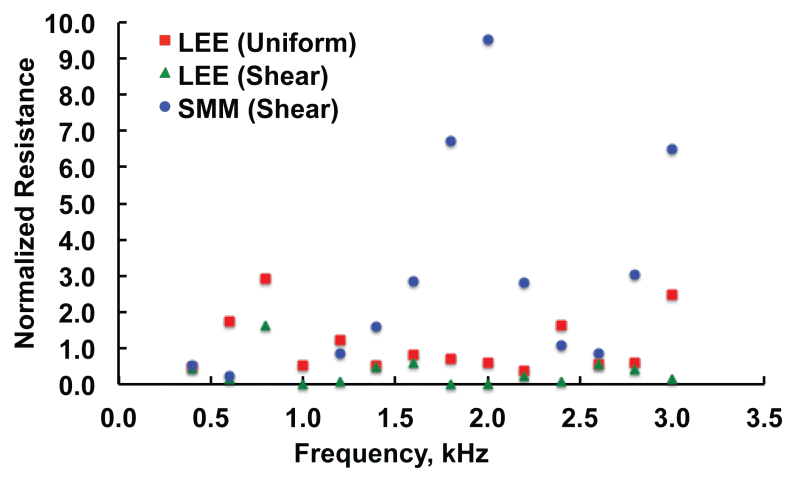

(c) Downstream source

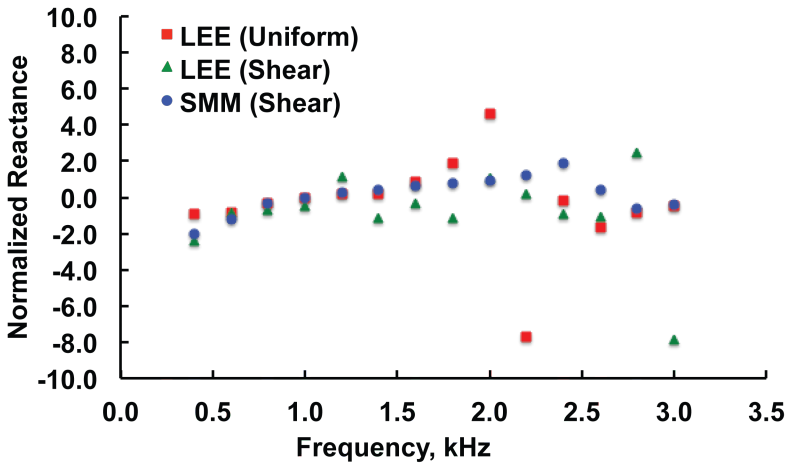

(b) Upstream source

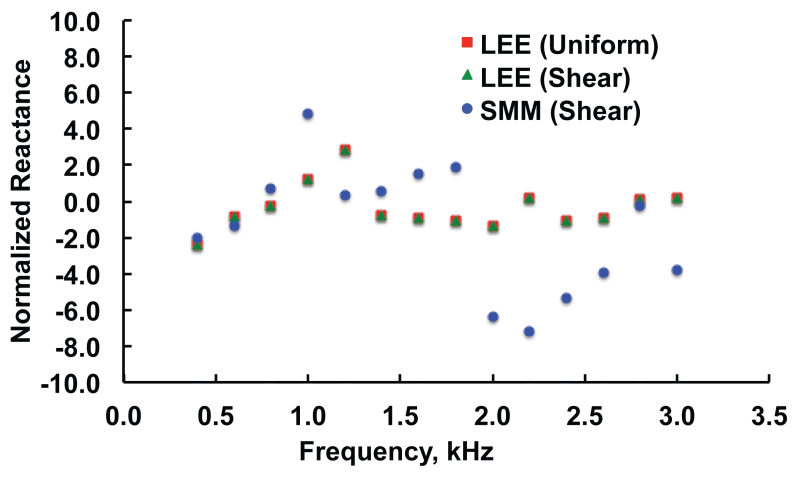

(d) Downstream source

Figure 17. Educed normalized impedance for the wire mesh liner at Mach 0.5 using measured data from the GFIT with mean shear. 